\title{
Regulatory networks in mechanotransduction reveal key genes in promoting cancer cell stemness and proliferation
}

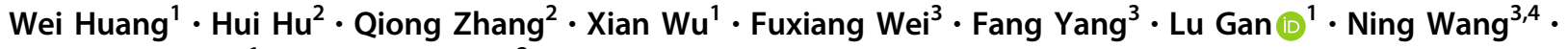 \\ Xiangliang Yang ${ }^{1} \cdot$ An-Yuan Guo $\mathbb{D}^{2}$
}

Received: 16 January 2019 / Revised: 21 June 2019 / Accepted: 8 July 2019 / Published online: 12 August 2019

(c) The Author(s) 2019. This article is published with open access

\begin{abstract}
Tumor-repopulating cells (TRCs) are cancer stem cell (CSC)-like cells with highly tumorigenic and self-renewing abilities, which were selected from tumor cells in soft three-dimensional (3D) fibrin gels with unidentified mechanisms. Here we evaluated the transcriptome alteration during TRCs generation in 3D culture and revealed that a variety of molecules related with integrin/membrane and stemness were continuously altered by mechanical environment. Some key regulators such as MYC/STAT3/hsa-miR-199a-5p, were changed in the TRCs generation. They regulated membrane genes and the downstream mechanotransduction pathways such as Hippo/WNT/TGF- $\beta /$ PI3K-AKT pathways, thus further affecting the expression of downstream cancer-related genes. By integrating networks for membrane proteins, the WNT pathway and cancer-related genes, we identified key molecules in the selection of TRCs, such as ATF4, SLC3A2, CCT3, and hsa-miR-199a-5p. Silencing ATF4 or CCT3 inhibited the selection and growth of TRCs whereas reduction of SLC3A2 or hsa-miR-199a-5p promoted TRCs growth. Further studies showed that CCT3 promoted cell proliferation and stemness in vitro, while its suppression inhibited TRCs-induced tumor formation. We also contemplated CCT3 as a stemness-related gene. Our findings provide insights in the mechanism of TRCs selection through transcriptome analysis.
\end{abstract}

\section{Introduction}

Cancer stem cells (CSCs) are a small subpopulation of cancer cells identified in both hematologic malignancies and solid tumor types with high tumorigenicity and high capabilities of self-renewal [1]. CSCs may be the source of

These authors contributed equally: Wei Huang, Hui $\mathrm{Hu}$

Supplementary information The online version of this article (https:// doi.org/10.1038/s41388-019-0925-0) contains supplementary material, which is available to authorized users.

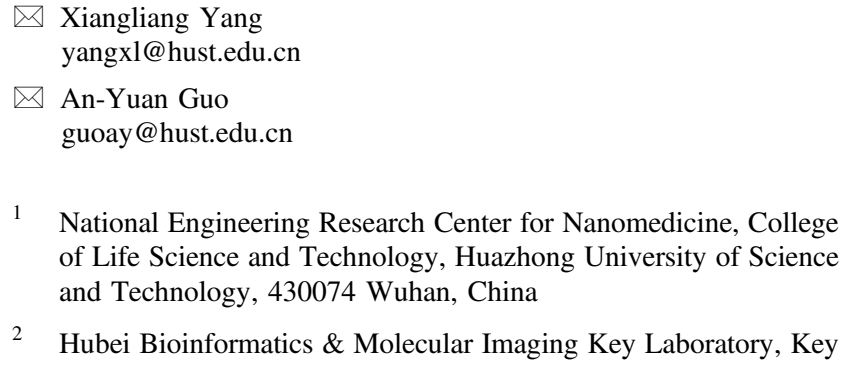

1 National Engineering Research Center for Nanomedicine, College of Life Science and Technology, Huazhong University of Science and Technology, 430074 Wuhan, China

2 Hubei Bioinformatics \& Molecular Imaging Key Laboratory, Key

tumor initiation, progression, metastasis, relapse, and resistance to chemotherapy [2]. Although there are several hypotheses about the CSCs origin, a lack of clear understanding about its origin limits the development of CSCtargeted therapy [3]. Our previous work has developed a mechanical method to select and/or generate CSC-like tumor-repopulating cells (TRCs) from cancer cells by culturing them in soft three-dimensional (3D) fibrin gels [4]. These TRCs were selected as highly self-renewing tumorigenic subpopulation [5] that is crucial to tumor initiation and progression [4, 6]. TRCs selected from B16 mice melanoma cells were able to initiate tumor in wild-

Laboratory of Molecular Biophysics of the Ministry of Education, College of Life Science and Technology, Huazhong University of Science and Technology, 430074 Wuhan, China

3 Department of Biomedical Engineering, College of Life Science and Technology, Huazhong University of Science and Technology, Wuhan 430074 Hubei, China

4 Department of Mechanical Science and Engineering, College of Engineering, University of Illinois at Urbana-Champaign, Urbana, IL 61801, USA 
type mice with as few as ten cells [4]. Cancer cells from several murine and human cell lines were able to be selected with the soft fibrin gel approach $[4,5,7]$. Thus, the process of TRCs selection/transformation is a very good model to study the origin, feature and mechanism of CSCs. Therefore, a comprehensive study on expression and regulation of the origin of TRCs may give us insights into the acquisition of cancer stemness and potential targets for prevention and treatment of cancer.

TRCs were selected or transformed by mechanotransduction under 3D soft gels and there are a few studies about it. The force transduction from extracellular matrix (ECM) to the cytoskeleton is mediated by integrins, focal adhesions, and mechanosensitive ion channels on the plasma membrane [8] and then activated mechanoresponsive-signaling elements, such as transforming protein RHOA [9]. Some well-known transcription regulators, such as $\beta$-catenin (WNT effector), YAP, and TAZ [10], are functionally required for eliciting cellular mechanoresponses. There are four well-studied classical mechanotransduction pathways Hippo/Wnt/TGF- $\beta / \mathrm{PI} 3 \mathrm{~K}-$ AKT [11], which may impact the stemness of cancers [12]. The self-renewing ability of TRCs was maintained by histone 3 lysine residue 9 (H3K9) demethylation and Sox2 gene expression [5]. However, the regulatory network on gene expression and key regulators under mechanotransduction of TRCs acquisition need to be uncovered.

Transcription factors (TFs) and miRNAs are two kinds of key regulators in gene regulatory network. They can regulate individually or co-regulate the same target gene to form a feedforward loop or feedback loop [13], which plays an important role in diseases and biological processes [14], including CSCs plasticity [15]. Several studies performed the transcriptome-profiling analysis for mechanical transduction in 3D co-cultured cardiomyocytes [16], while few such study on TRCs was conducted. The transcriptome profiling and regulatory networks of the TRCs selection process could provide a comprehensive landscape to the underlying molecular and regulatory mechanisms and provide new insights to cancer stemness.

In this study, to better understand the TRCs selection and transformation, we sequenced the transcriptome and miRNA for samples that collected during the soft fibrin gels selection process, followed by systematical bioinformatics analysis on expression and regulation. We used HeLa cells, the earliest human cancer-derived cell line established as a model for studying cancer in culture [17], to examine how HeLa TRCs might differ from those unselected HeLa cells. We identified and validated a few key regulators and genes, which were important in the TRCs selection process. It showed a comprehensive view of the gene expression and regulation for TRCs selection and provided potential targets for cancer therapy.

\section{Results}

\section{Dynamic changes of gene expression in TRCs selection by $3 \mathrm{D}$ soft fibrin gels}

To obtain and study the TRCs, we seeded the human cervical carcinoma HeLa cells into different stiffness fibrin gels (90, 420, and $1050 \mathrm{~Pa}$ ). HeLa cells grew into round multicellular tumor spheroid from day 1 to day 7 (Fig. 1a, Supplementary Fig S1). HeLa cells cultured in $90 \mathrm{~Pa}$ fibrin gels (HeLa TRCs) had significant more colony numbers from day 1 and bigger colony size from day 3 than in the stiffer ones (420 or 1050 Pa) (Fig. 1b, c). Transwell assays showed that HeLa TRCs had appreciably higher migration and invasion ratios than HeLa cells cultured on rigid plastic (HeLa 2D cells) (Fig. 1d). Expressions of well-known CSCs-related genes SOX2/NANOG/ZFX/OCT4 (POU5F1) [18] were all significantly increased in TRCs compared with HeLa 2D cells at day 3 (Fig. 1e). SOX2 and NANOG expression were significantly increased from day 1-3 (Supplementary Fig. S2). Together, these findings suggest that HeLa TRCs in 3D soft fibrin gels are unique in promoting multicellular tumor spheroid formation and growth with enhanced self-renewing capacities.

To study the dynamic changes of gene expression and regulatory mechanisms under TRCs selection and transformation, we sequenced the HeLa 2D cells (as $0 \mathrm{~h}$ ) and HeLa 3D TRCs at four time points: $12 \mathrm{~h}, 1$ day, 3 day, and 5 day (Supplementary Fig. S3). As a result, high expressed genes in 2D cells were much more than 3D TRCs (Supplementary Fig. S4A). Then, we conducted pairwise comparison analyses among the five samples and filtered out 1305 differentially expressed genes (DEGs) including 28 TFs (Fig. 1f) and 118 differentially expressed miRNAs (DEMs) (Supplementary Fig. S4B, C). About $81 \%$ of these DEGs were in the comparison of $12 \mathrm{~h}$ vs $0 \mathrm{~h}$ and most of them (97.5\%) were downregulated (Supplementary Fig. S4D). This indicates that many genes were significantly altered as the mechanical environment changes by switching from rigid 2D plastic dishes to soft 3D gels. However, only 12 DEMs were changed from $2 \mathrm{D}$ to $3 \mathrm{D}$ gels, which were much less than the DEMs in comparisons of 3 day vs $0 \mathrm{~h}$ (62 DEMs) or 5 day vs 3 day (61 DEMs) (Supplementary Fig. S4C, D). We defined genes different between $12 \mathrm{~h}$ and $0 \mathrm{~h}$ as potential mechanicalrelated genes since the mechanical forces changed. We collected 144 integrin and focal adhesion related membrane protein genes and found only a small part of them were DEGs (Supplementary Fig. S4E). Analyses revealed 394 genes and 49 miRNAs, whose expression was continuously increased or decreased from $12 \mathrm{~h}$ to 3 day. Among them, 25 genes and 8 miRNAs were reported to be associated with tumor growth and proliferation (Fig. 1g, Supplementary Fig. S4E). In further analysis, we focused on these different genes, 28 
A

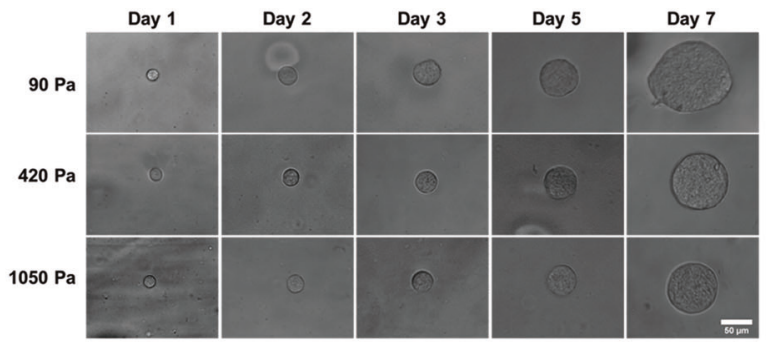

B
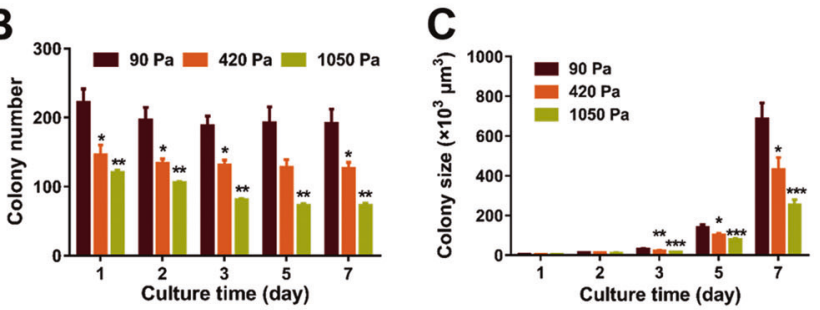

$\mathbf{F}$

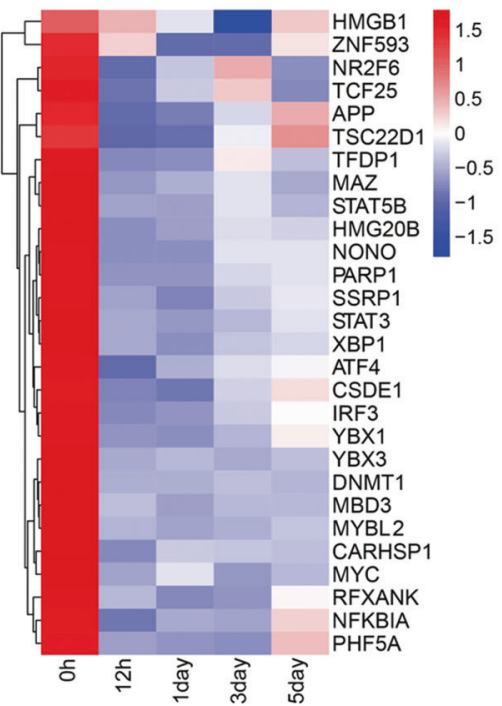

Fig. 1 Matrix softness enhances stemness genes in HeLa TRCs and differentially expressed TFs/miRNAs. a Representative images of a single HeLa cell growing into a multicellular tumor spheroid in different stiffnesses $(90,420$, or $1050 \mathrm{~Pa}) 3 \mathrm{D}$ fibrin gels cultured from day 1 to day 7. Scale bar, $50 \mu \mathrm{m}$. b, c Colony number and size of HeLa TRCs in different 3D gels with culture time from day 1 to day 7. d TRCs (90-Pa 3D gels) perform higher invasion and migration ability than HeLa 2D. Representative images (left) and quantification of ten

differentially expressed TFs and 8 cancer related continuously changed miRNAs to construct regulatory network (Supplementary Fig. S3).

\section{Membrane protein genes were significantly affected by mechanotransduction}

Previous studies have shown that membrane proteins play vital roles in mechanotransduction [8]. Gene ontology (GO) and the pathway enrichment analyses for the 1305 DEGs (Fig. 2a) showed that they were significantly enriched in

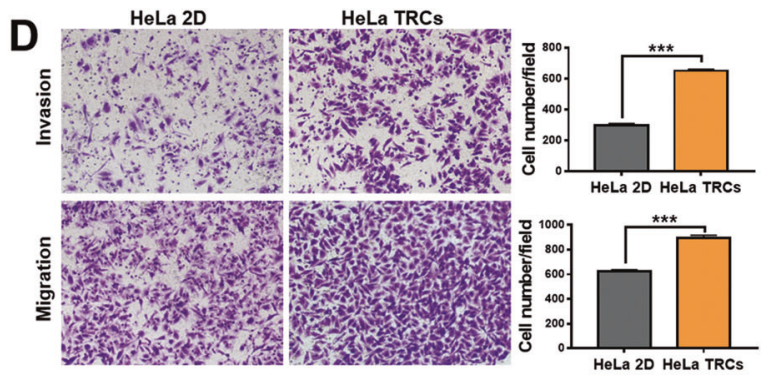

E

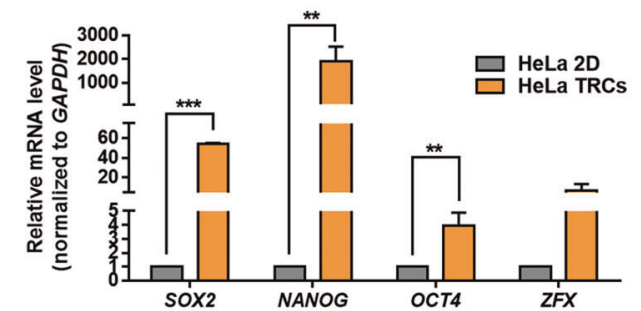

G

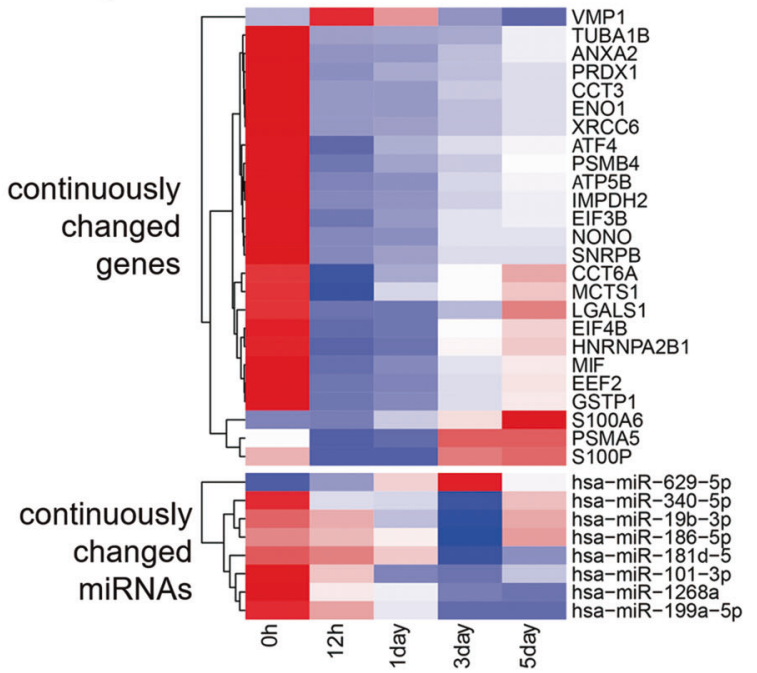

randomly selected fields (right) are shown. e Expressions of stem cell markers in HeLa 2D and HeLa TRCs (90 Pa, day 3) were quantified by real-time PCR. f Heatmap of differentially expressed TFs, scale from blue to red indicates the expression level from low to high. g Heatmap of continuously changed genes and miRNAs. Mean \pm s.e.m., $n=3$ independent experiments except for $\mathbf{c}$ where $n=10$. Asterisks in $\mathbf{b}$ and c are compared with 90-Pa group. $* p<0.05,{ }^{* *} p<0.01, * * * p<0.001$; ns, not significantly different (Student's $t$ test)

mechanotransduction related terms such as membrane (FDR: 4.45E-51), ECM (1.67E-13), and focal adhesion (7.75E-23). These results highlighted potential roles of membrane protein genes in the TRCs generation under mechanotransduction. There were 25 integrin and cell/focal adhesion related membrane genes significantly changed from 2D to 3D gels (Fig. 2b) and part of those genes were continuously increased in the $3 \mathrm{D}$ gels from $12 \mathrm{~h}$ to 3 day, such as ANXA2, ADAM15, ITGA3, ITGB5, SLC3A2, and GRB2. To explore the regulation of these membrane DEGs, a TF and miRNA co-regulatory network for them were 


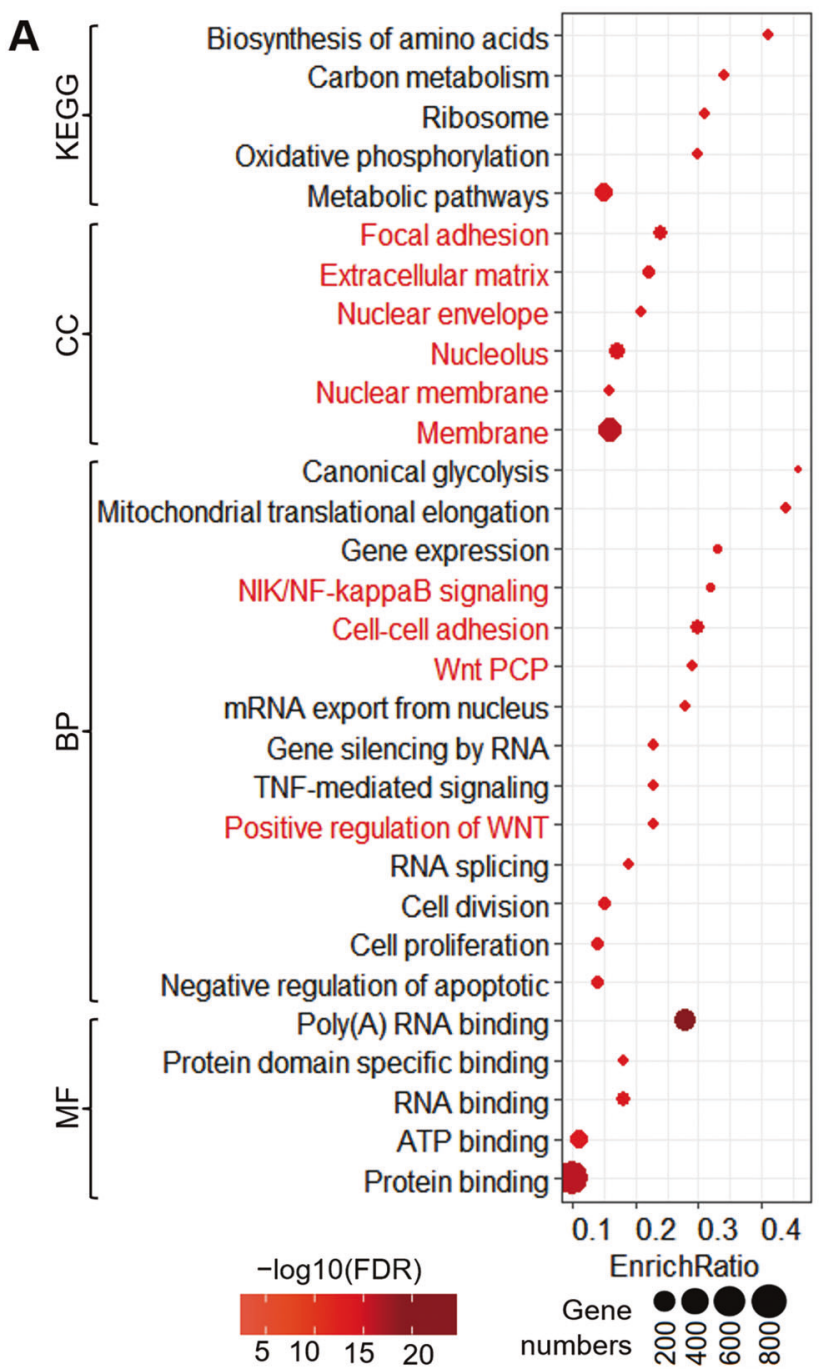

Fig. 2 Functional enrichment in the 3D fibrin gels culture and regulatory networks for membrane. a Top 30 significantly enriched terms $(p<1.0 \mathrm{E}-4)$ in Gene Ontology and the KEGG pathway for DEGs. Terms in red font are related to mechanotransduction or cancer proliferation. EnrichRatio The ratio of enriched genes in the term. FDR false discovery rate. CC cellular component. BP biological process. MF molecular function. b Heatmap of 25 differentially expressed

constructed. Membrane genes were predicted to be regulated by nine differentially expressed TFs and five miRNAs (Fig. 2c). TFs MYC and STAT3 regulated nine and eight membrane genes, respectively. GRB2 is involved in the formation of focal adhesion complexes [19] and was regulated by STAT3 and IRF3. ITGA3, an integrin receptor for fibronectin, laminin, and collagen [20], was a direct target of hsa-miR-199a-5p. Conversely, membrane genes can also regulate TFs, for instance, GRB2 can inhibit the STAT3 activity [21] while ANXA2 positively regulate STAT3 [22]. These results indicated membrane genes may be regulated by miRNAs or TFs during the mechanotransduction.
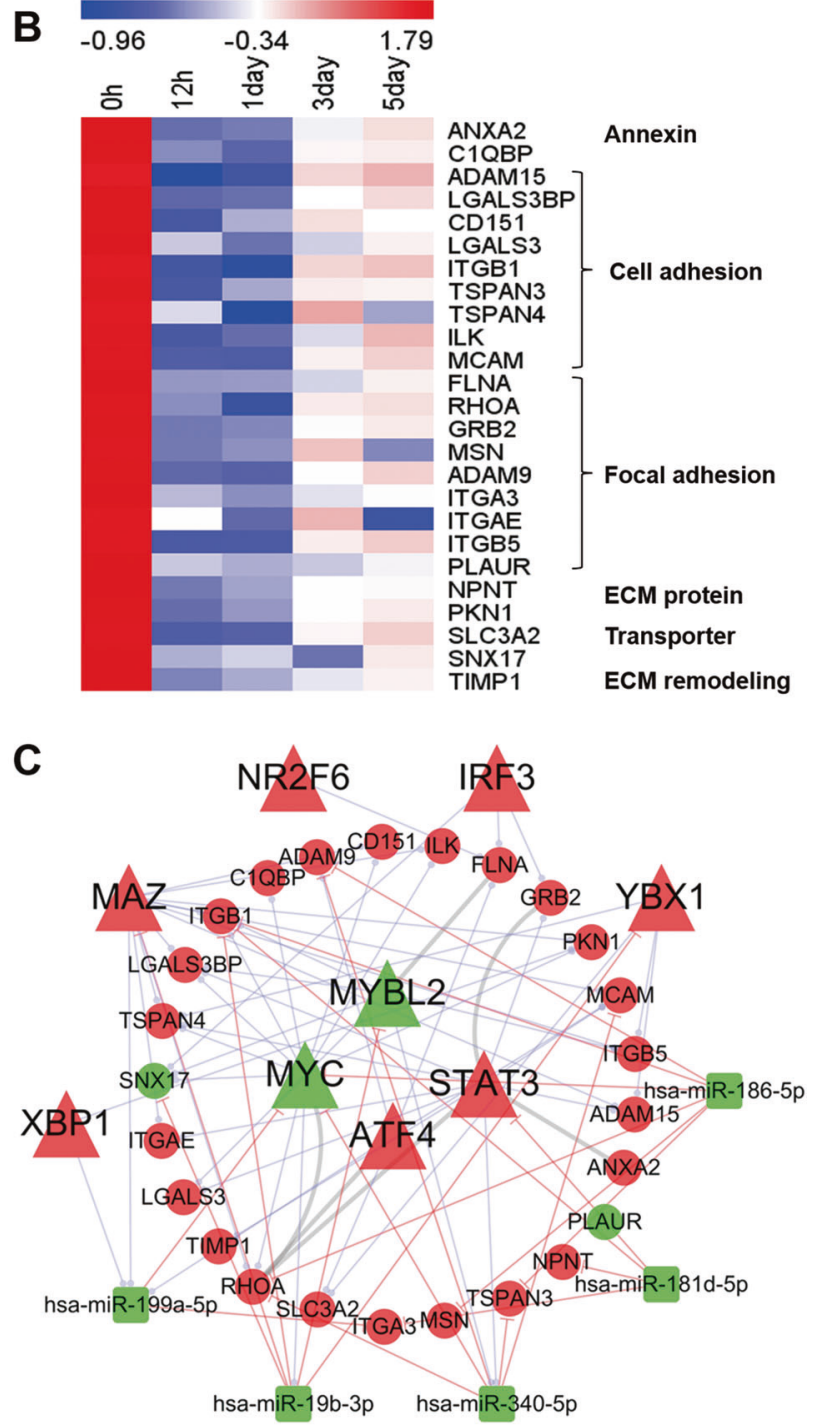

membrane protein genes. $\mathbf{c}$ TF and miRNA regulatory network for membrane proteins. Red and green nodes represent up and down regulations in comparison of 3 day versus $12 \mathrm{~h}$. Round nodes are genes, triangle nodes are TFs, and square nodes are miRNAs. Red edge represents miRNA regulation and purple edge indicates TF regulation. Gray edge represents membrane protein regulation

\section{Core regulators responsible for the changes of WNT/ Hippo/TGF- $\beta$ /PI3K-AKT pathways during TRCs process}

The mechanical stress in 3D gels will transfer through membrane to cytoplasm and alter the downstream signaling pathways. In the above analysis, we identified 27 DEGs were enriched in the positive regulation of WNT (8.93E-4) and the Wnt/planar cell polarity (WNT/PCP) pathway (2.46E-6) (Fig. 2a). WNT together with other three pathways (Hippo, PI3K-AKT, and TGF- $\beta$ ) were considered as classical mechanotransduction pathways [11]. Our results 
demonstrated that numerous DEGs were involved in the four pathways: Hippo (10\% of the 155 genes are DEGs), PI3K-AKT (9\% of 343), TGF- $\beta$ (13\% of 85 ), and WNT ( $8 \%$ of 143) (Fig. 3a). Most DEGs in the four pathways were upregulated from $12 \mathrm{~h}$ to 3 day during the $3 \mathrm{D}$ cultured (Fig. 3a). Interestingly, most TFs and miRNAs in the regulatory network of membrane genes (Fig. 2c) were also the common regulators in regulatory networks of these four mechanotransduction pathways (Fig. 3b). Seven TFs were significantly differentially expressed in the comparison of $12 \mathrm{~h}$ vs $0 \mathrm{~h}$ sample (Fig. 3a) and ATF4/STAT3/MYC have been reported to be associated with mechanotransduction or
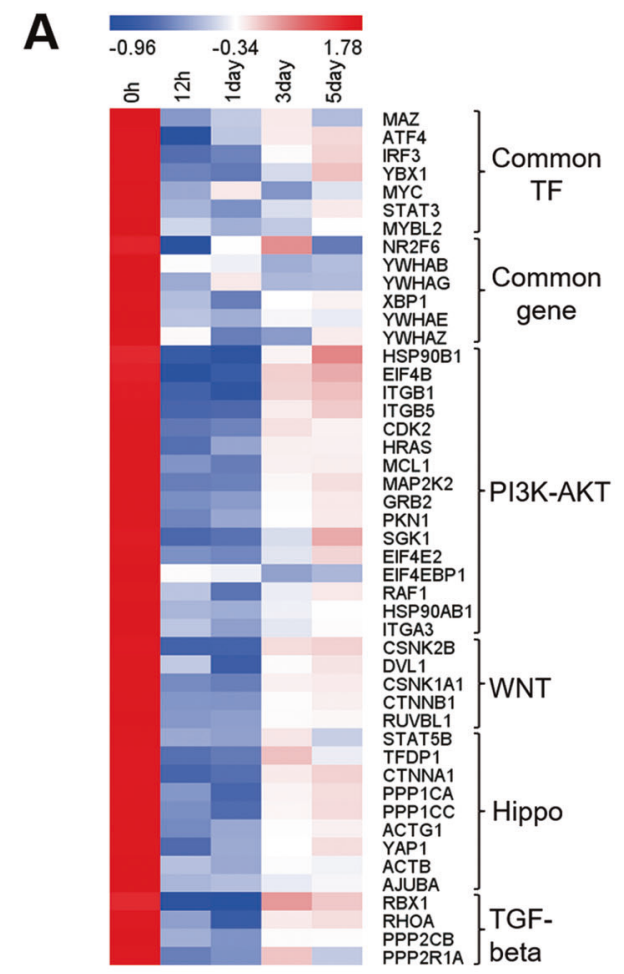

B
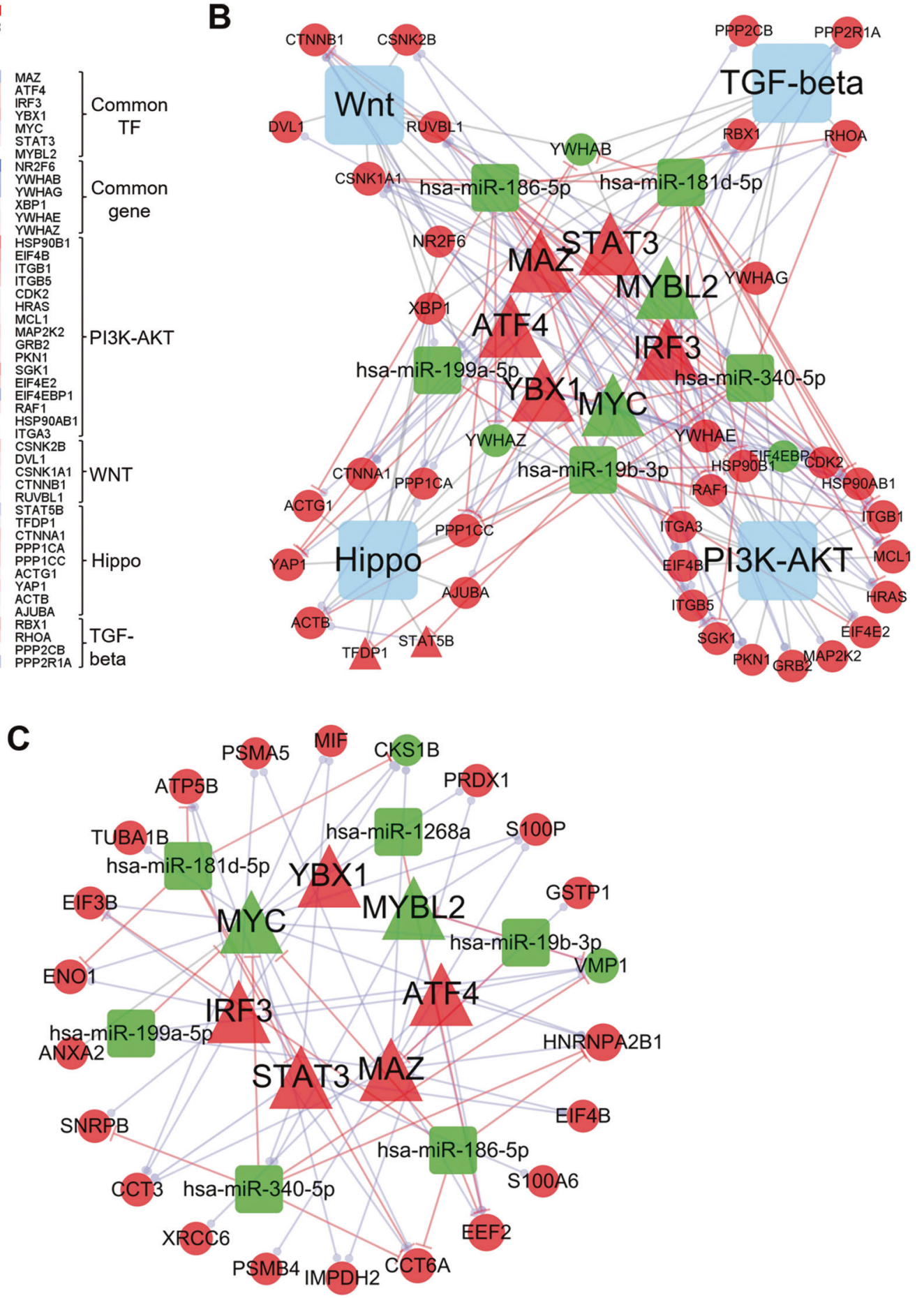

Fig. 3 Expression and regulation of genes in four classical pathways. a Expression heatmap of DEGs in four classical pathways, which are canonical Hippo, Wnt, TGF-beta, and PI3K-AKT pathways. b
Common regulators of the four pathways. Gray edge indicates the TFs/ miRNA regulates the corresponding pathway. $\mathbf{c}$ Regulation network of cancer-related genes 
activated under mechanical force [23, 24]. Meanwhile, miRNAs were continuously decreasing from $12 \mathrm{~h}$ to 3 day and some were reported to inhibit tumorigenesis induced by CSCs such as miR-186-5p/199a-5p [25, 26]. These results showed that these TFs and miRNAs were core regulators in the four classical mechanical signaling pathways.

\section{Continuously changed cancer-related genes were regulated by TFs and miRNAs}

The mechanical stress transferred from membrane to downstream pathways, and then affected the expressions of downstream genes. For the affected downstream genes, we focused on the 25 continuously changed cancer-related DEGs (Fig. 1g). Among them, 23 were regulated by TFs and miRNAs (Fig. 3c). In the network, most genes and TFs were continuously increased while miRNAs were continuously decreased from $12 \mathrm{~h}$ to 3 day (Fig. $1 \mathrm{~g}$ ). We found that MYC as a core regulator in the network regulated half of the cancer-related genes (12/23). CCT3 was regulated by the largest number of TFs such as STAT3/MYC/MAZ/ IRF3. CCT3 promotes the proliferation of hepatocellular carcinoma [27]. These results suggested that TFs/miRNAs may be through the four mechanical pathways to directly or indirectly regulated cancer-related genes and thus influence the selection of TRCs.

\section{Enrichment analyses and integrated regulatory network revealed key regulators}

Previous studies have shown that the WNT pathway plays important roles in mechanotransduction [11] and our enrichment results also revealed the activation of the WNT pathway (Fig. 3b). Next, we performed pathway enrichment analysis $(P<0.01$, Bonferroni) (Fig. 4a) for the DEGs in the WNT pathway network (Fig. 3b), membrane proteins (Fig. 2b), and cancer-related genes (Fig. 3c) to identify their pathway crosstalk. Results showed many crosstalk genes between multiple pathways. For instance, CTNNB1 and RHOA participated in WNT and focal adhesion pathways (Fig. 4a), as well as cancer-related pathways. Membrane genes such as ITGB1/ITGB5/ITGA3 were involved in both focal adhesion and WNT pathways (Fig. 4a).

To identify more candidate regulators or genes in the TRCs selection for experimental validation, we constructed a combined network (Fig. 4b) for extending Fig. 4a. As a result, we found nine regulators (five TFs and four miRNAs) act as crosstalk regulators involved in the regulation of membrane proteins, WNT pathways, and cancer-related genes. For example, as a key crosstalk regulator in the network, STAT3 can regulate cancer gene CCT3, membrane gene ITGAE, and the WNT pathway gene CCNTBI. In addition, we also found many genes in our network that were not available in Fig. 4a, such as $C C T 3$ and $C K S 1 B$. Almost no studies reported the cancer-related gene CCT3 in the TRCs-screening process or CSCs, while it may play important roles in our study. These analyses provided evidence that genes or regulators may play vital roles in the crosstalk between mechanotransduction and cancer proliferation regulation.

\section{Key genes and miRNA impacting selection of TRCs}

Our transcriptome analysis indicated that many TFs, miRNAs, and genes involved in multiple pathways to mediate mechanotransduction and influence the selection of TRCs. To verify the function of genes in the 3D culture process and TRCs selection, firstly, we validated the expression of hsa-miR-199a-5p in HeLa TRCs at 3 day and HeLa 2D cells as control (Supplementary Fig. S5A). Herein, colony formation of HeLa TRCs was significantly decreased with hsa-miR-199a-5p mimic. This suggested that the selection process of HeLa TRCs was inhibited by hsa-miR-199a-5p (Fig. 4c-e), which was reported to inhibit the engraftment and repopulation capacity in liver [28]. Previous studies have shown the regulation of ITGA3 by hsa-miR-199a-5p inhibits cancer cell migration and invasion [29]. Our experiments showed that ectopic expression of hsa-miR-199a-5p led to downregulation of ITGA3 and this may reduce 3D colony formation (Supplementary Fig. S5B).

Next, results showed that the expression of TFs (ATF4 and STAT3) and genes (FLNA, RHOA, ITGA3, CCT3, $C K S 1 B$, and $S L C 3 A 2)$ were changed significantly from HeLa 2D on rigid dish to HeLa TRCs in 3D soft fibrin gels (Fig. 4f). Further, after transfected siRNAs for $24 \mathrm{~h}$, the TF ATF4 significantly suppressed the colony growth and number (Fig. 4g), which was probably through inhibition of NANOG and OCT4 expression (Supplementary Fig. S5C). Transfection of CCT3 siRNA also significantly reduced colony number and size (Fig. 4g). But the silence of SLC3A2 increased the colony number and size (Fig. 4g). These results suggested that ATF4 and CCT3 promoted the selection of HeLa TRCs, while SLC 3A2 suppressed it.

\section{CCT3 promoted cell proliferation and stemness in vitro, while its suppression inhibited TRCs- induced tumor formation}

To further investigate the function of CCT3 in cancer, we constructed pcDNA 3.1-CCT3 plasmid to overexpressing CCT3 and silencing CCT3 with siRNA and shRNA. The transfection of pcDNA 3.1-CCT3 or CCT3 RNAi significantly induces or reduces CCT3 expression detected by immunofluorescence (Supplementary Fig. S6A). Then we sequenced the mRNAs of samples of CCT3 overexpression, 
A

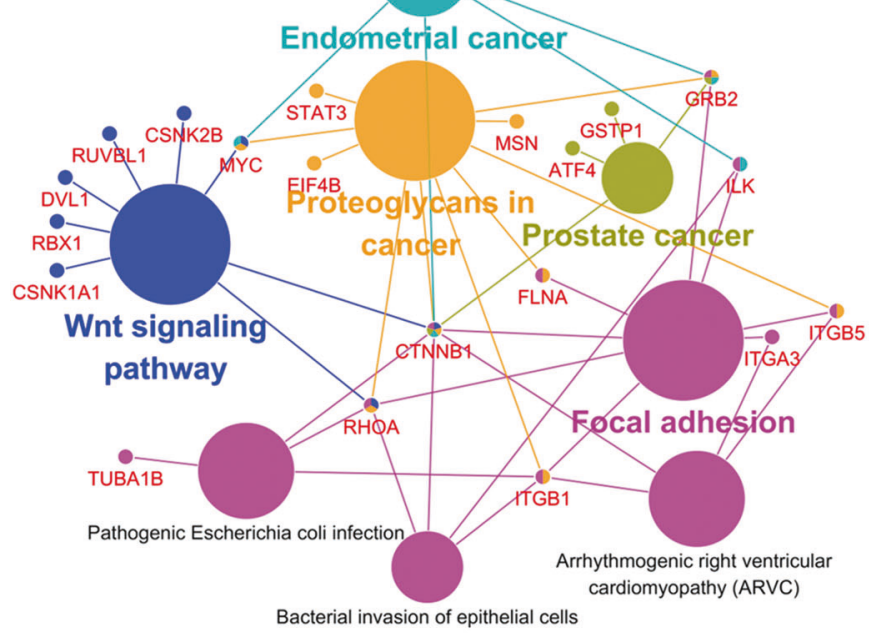

C

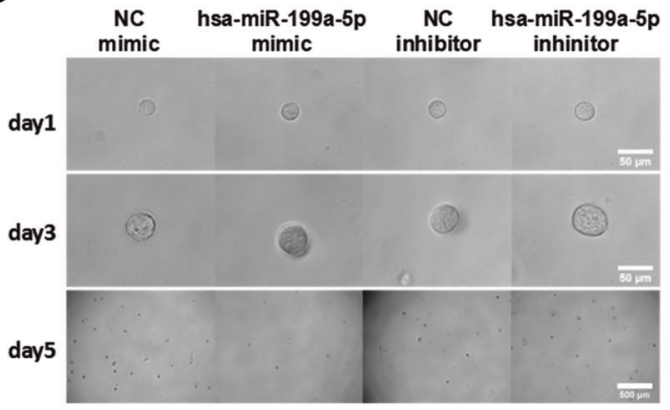

B

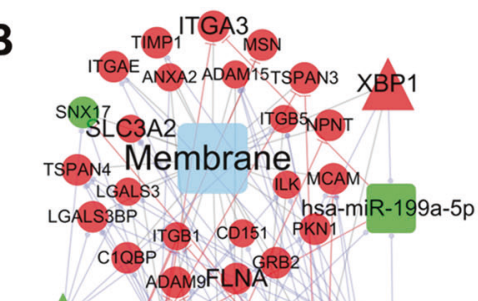

MYBL2 MYC ATF 4 STAT3

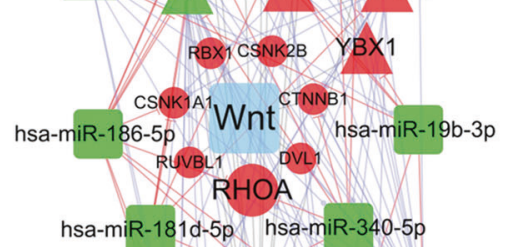

S100AE CETGA
hsa-miR-1268a IMPPA2B1 MAZ ATPB CCT3 MAZ

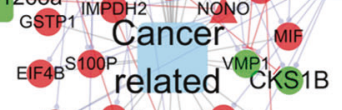

PRDX1 XRCC6 PSMA5

TUBB⿴囗十⺝

$\mathbf{F}$

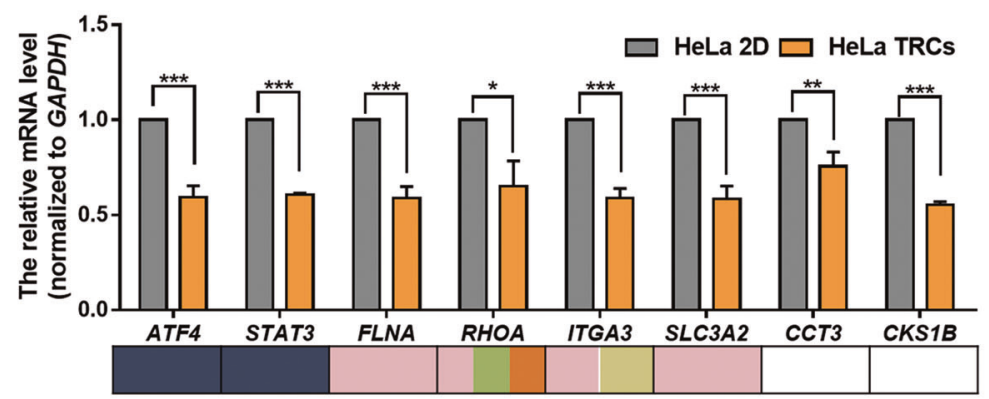

D

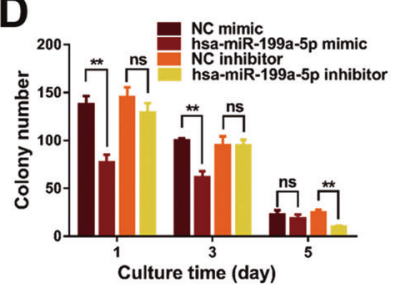

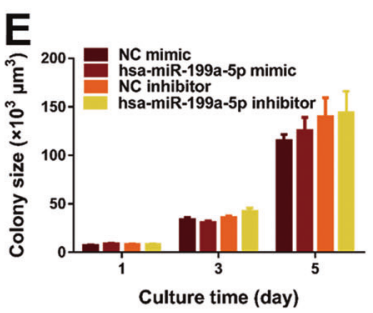

G
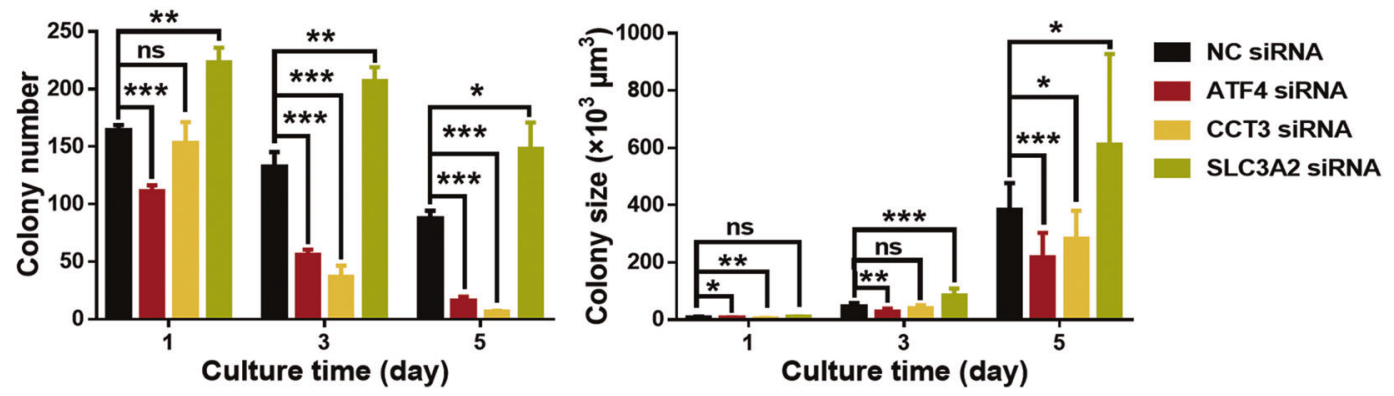

CCT3 RNAi and negative control (NC) HeLa cells and filtered out 260 or 520 DEGs (Fig. 5a) when compared CCT3 overexpression or CCT3 RNAi with NC. A large number of upregulated genes after CCT3 overexpressed were significantly enriched in cell proliferation-related pathways such as cell cycle whereas downregulated genes 
Fig. 4 Integrated regulatory network and ATF4, CCT3, SLC3A2, and hsa-miR-199a-5p significantly impact TRCs selection. a Pathway enrichment results shown as a functionally grouped network by ClueGO. Functionally related terms are displayed using the same color. Crosstalk genes involved in multiple pathways are proportionally marked with the same color as the related pathway. b An integrated network of networks from membrane protein genes, the WNT pathway and cancer genes. c Images of HeLa cells in 90-Pa fibrin gel after infected with hsa-miR-199a-5p inhibitor or mimic. Scale bar, $50 \mu \mathrm{m}$ (top); $500 \mu \mathrm{m}$ (bottom). d, e Colony numbers (d) and sizes (e) affected by hsa-miR-199a-5p mimic or inhibitor at day 1 and day 3 . $\mathbf{f}$ Expression of selected genes quantified by real-time PCR in HeLa TRCs (90 Pa fibrin gel at day 3 ) and HeLa 2D cells. g The colony number and size were counted at days 1,3 , and 5 for the silence of ATF4, CCT3, and SLC3A2. NC siRNA is negative control siRNA. Mean \pm s.e.m., $n=3$ independent experiments except for colony size of $\mathbf{e}$ and $\mathbf{g}$ where $n=10 . * p<0.05, * * p<0.01, * * * p<0.001$; ns not significantly different (Student's $t$ test)

enriched in the metabolic pathway (Fig. 5b, Supplementary Fig. S7), implying that CCT3 may participate in the cell cycle pathway to promote cancer proliferation. To verify the cancer proliferation ability of CCT3, we used HeLa cell and Bel7402 cell. Overexpression of CCT3 promoted cell proliferation significantly and its silencing suppressed proliferation. Cotransfected both pcDNA 3.1-CCT3 and CCT3 siRNA silenced the promoting proliferation ability of CCT3. (Fig. 5c, d, left). These showed CCT3-promoted proliferation in $2 \mathrm{D}$ cell lines. Then we seeded these transfected cells into $90 \mathrm{~Pa}$ fibrin gels to study what will happen in 3D. We calculated both colony number and colony size of HeLa TRCs and Bel7402 TRCs at day 1 and day 3 (Fig. $5 \mathrm{c}, \mathrm{d}$, middle and right). Colony size of these TRCs had no significant difference, but colony numbers were significantly impacted. With CCT3 overexpression, colony numbers changed from 22 to 97 at day 1 in HeLa TRCs, which means the TRCs ratio significantly enhanced with CCT3 overexpression. Silencing CCT3 in the process markedly decreased the TRCs colony numbers. These suggest that CCT3 is indispensable for proliferation of HeLa and Bel7402 TRCs.

Is CCT3 important in the other cancer types? We turned to the cancer genome data (TCGA) and found that CCT3 was significantly upregulated in 12 cancer types (BLCA, BRCA, LUAD, etc.) of 14 cancer types with paired cancer and adjacent samples (Fig. 5e). High level of CCT3 was significantly associated with poor prognosis in patients of six cancers such as liver cancer (LIHC) and kidney cancer (KIRP) (Fig. 5f, Supplementary Fig. S6B), which was consistent with the previous reports [27, 30]. Taken together, these results suggest a cancer-promoting role of CCT3 in different kinds of cancers.

After verifying the CCT3 functions in promoting cell proliferation and TRCs selection, we further investigated whether CCT3 is related to cancer cell stemness or not.
Results of stemness enrichment analysis for DEGs from CCT3 overexpression or silencing separately showed that they were both enriched in stemness-related terms such as embryonic stem cells and E2F4 target gene sets etc. (Fig. 6a). Besides, the results of side population (SP) cells (stem-like cell population) analysis showed that there were more SP cells $(3.85 \%)$ in CCT3 overexpression cells, but less $(0.14 \%)$ in shCCT3 cells (Fig. 6b). The mRNA levels of some reported cancer stemness markers like CD133, ETV4, E2F4, and KRT8 were upregulated with CCT3 overexpression (Fig. 6c). CD133, KRT8, and ZFX were downregulated with CCT3 silencing. And their proteins were in higher expression in both HeLa and Bel7402 TRCs than their 2D cells, which were consistent with CCT3 protein (Fig. 6d). Next, we constructed a subcutaneous xenotransplantation tumor model to study the CCT3 role in tumorigenesis in vivo (Fig. 6e-g). TRCs showed higher tumorigenesis than 2D cells, but TRCs + shCCT3 reduced the TRCs tumorigenesis ability. Low levels of CCT3 inhibited TRCs proliferation in vivo. All these suggest that CCT3 is a cancer stemness-related gene.

\section{Discussion}

Many studies have shown that matrix and cell adhesion will change cell phenotype. Previous studies have reported that matrix softness such as soft 3D cell culture has substantial impacts on cell stemness [5]. However, how the matrix stress influences the gene expression and regulation is unclear. In this study, we applied high-throughput sequencing and regulatory network analysis, followed by experimental validation to explore transcriptome changes and the regulatory mechanism of matrix mechanic-induced TRC formation in cancer cells. We demonstrated that the ECM affects membrane proteins by force and lead to the change of downstream TFs, which regulate downstream WNT/ TGF- $\beta / \mathrm{PI} 3 \mathrm{~K}-\mathrm{AKT}$ pathways to affect many cancer-related genes and affect the selection of TRCs (Fig. 7). We have identified and validated several molecules including $\mathrm{TF}$ ATF4, transmembrane protein SLC3A2, a potential new stemness-related gene CCT3, and hsa-miR-199a-5p, which are important in mechanotransduction and affect TRCs selection process.

Our transcriptome results revealed that many genes were significantly changed when cells were transferred from $2 \mathrm{D}$ rigid to $3 \mathrm{D}$ soft environment (expression levels of experimentally validated genes and miRNA in sequencing data were shown in Supplementary Table S1). Functional analysis showed that many DEGs were enriched in the WNT pathway and the WNT/PCP subpathway. A key WNT signal transduction molecule DVL1 [31] and its downstream-activated molecular switch, small GTPase 

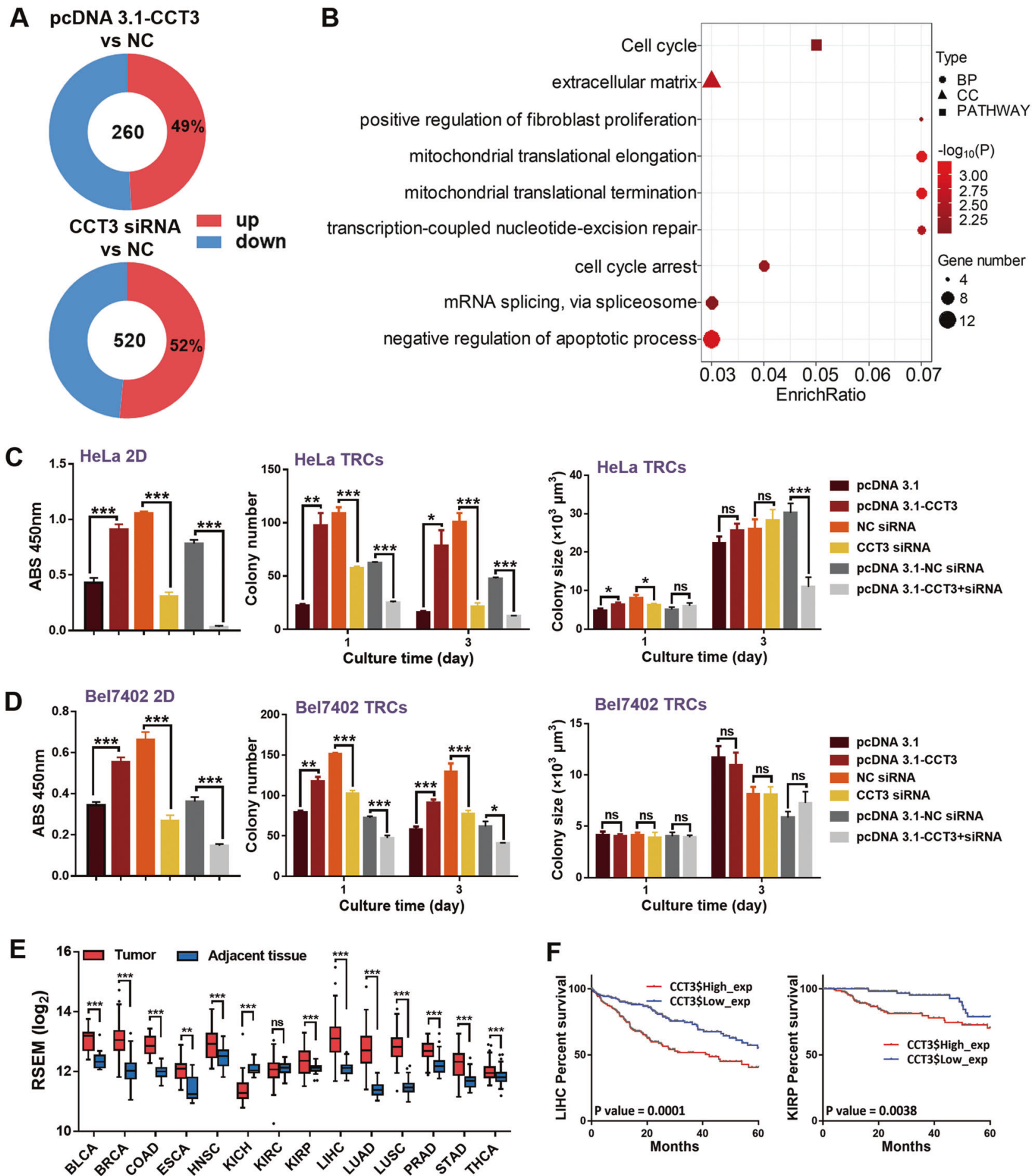

Fig. 5 CCT3 promotes selection of TRCs and proliferation of tumor. a Summary of DEGs by overexpressing or silencing CCT3. b Significantly enriched terms $(p<0.01)$ in Gene Ontology and the KEGG pathway after CCT3 overexpression. c, $\mathbf{d}$ CCT3 promotes proliferative activity of both 2D cells and TRCs in HeLa and Bel7402 cell lines. HeLa and Bel7402 2D cells were cultured $48 \mathrm{~h}$ after transfected with vectors. Colony numbers (middle) and colony sizes (right) were

RHOA [32], were both continuously increased from $12 \mathrm{~h}$ to 3 days (Fig. 3a). The activation of $R H O A$ could promote the counted at day 1 and day 3. Mean \pm s.e.m., $n=3$ independent experiments except for $\mathbf{c}$, d left panel where $n=10$. e Boxplot of CCT3 expression in 14 cancers with paired adjacent samples from TCGA. f Kaplan-Meier survival curve for overall survival (5 years) of patients in liver and kidney cancers according to CCT3 expression status (high or low expression, divided by median). $* p<0.05, * * p<$ $0.01, * * * p<0.001 ;$ ns not significantly different (Student's $t$ test)

expression of Rho kinase (ROCK), then regulate the cytoskeleton to transmit the received mechanical stimuli to 

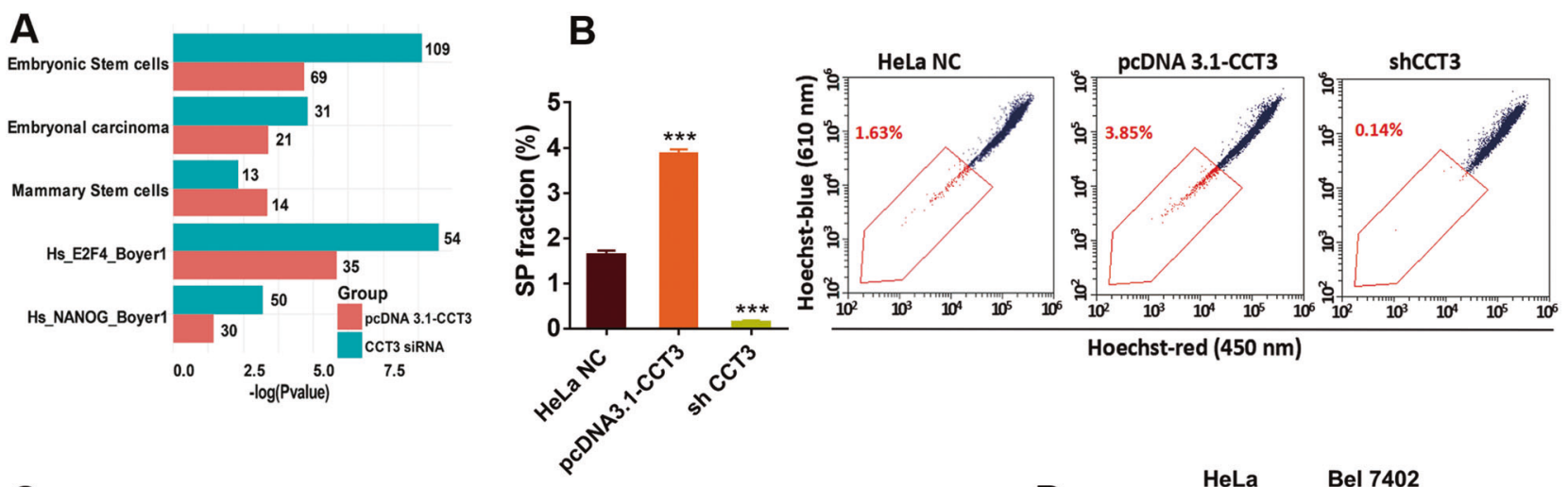

C

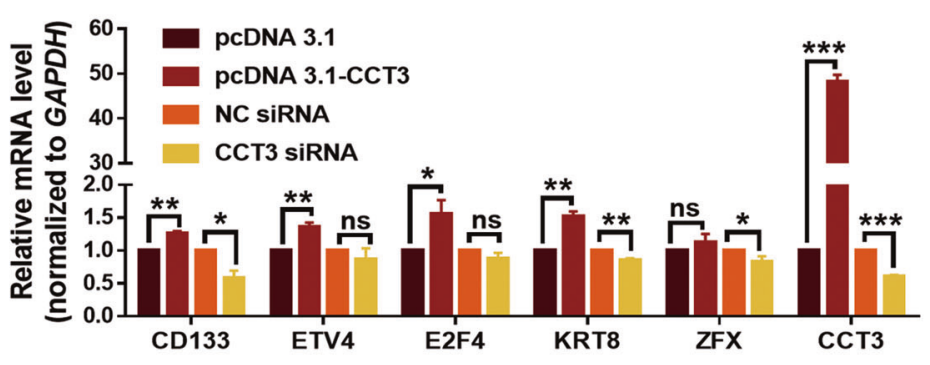

E

\begin{tabular}{lc}
\multicolumn{2}{c}{$\begin{array}{c}\text { Subcutaneous xenotransplanted } \\
\text { tumor in BALB/c Nude mice }\end{array}$} \\
\hline $5 \times 10^{5}$ HeLa cells & Tumor formation \\
\hline $2 \mathrm{D}$ cells & $0 / 8$ \\
TRCs & $3 / 8$ \\
TRCs + shCCT3 & $2 / 8$ \\
\hline & \\
\hline $5 \times 10^{5}$ Bel7402 cells & Tumor formation \\
\hline $2 \mathrm{D}$ cells & $0 / 8$ \\
TRCs & $3 / 8$ \\
TRCs + shCCT3 & $0 / 8$ \\
\hline
\end{tabular}

$\mathbf{F}$
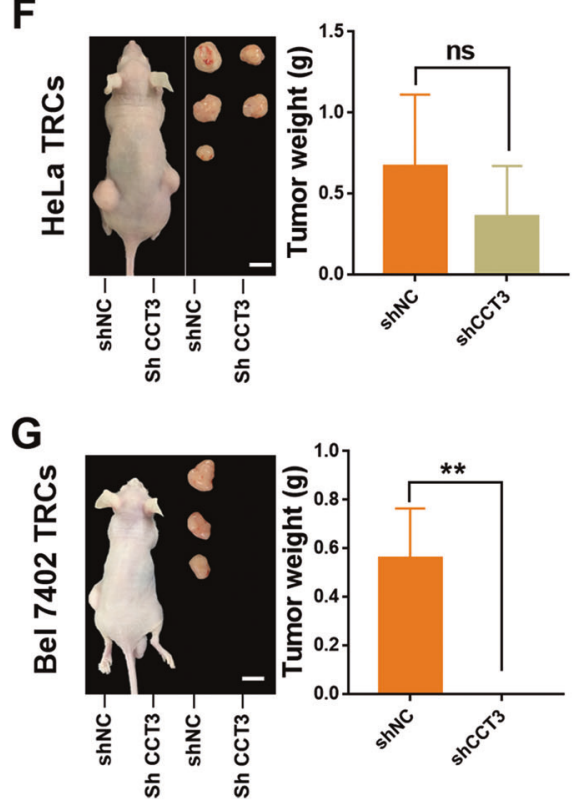

D

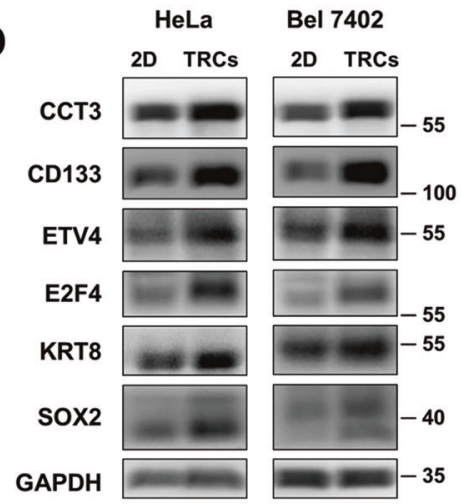

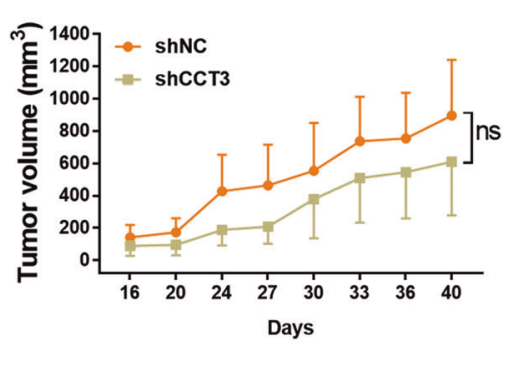

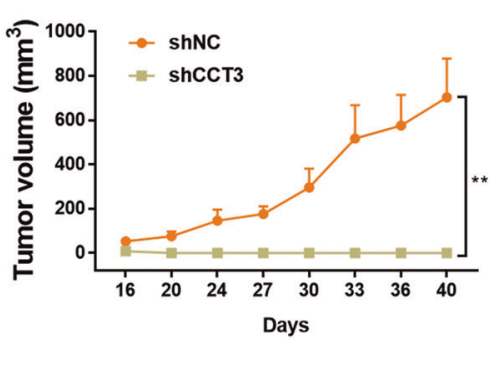

fibrin gels for 5 days, TRCs + shCCT3 were TRCs transfected with shCCT3 for $12 \mathrm{~h}$. They were then subcutaneously xenotransplanted into $\mathrm{BALB} / \mathrm{c}$ nude mice with 50,000 cells per mice. The tumor formation ratios were recorded after 40 days subcutaneous injection. $\mathbf{f}, \mathbf{g}$ Silence of CCT3-inhibited tumor formation ability of HeLa and Bel7402 TRCs. A total of $5 \times 10^{5}$ TRCs with scramble shRNA transfected were subcutaneous injected into left posterior subcutaneous of BALB/c nude mice, and TRCs transfected with CCT3 shRNA transfected were injected in the other side. Representative photos of tumor-bearing mice were shown (left), bar $=1 \mathrm{~cm}$. Data are represented as the mean \pm s.e.m. of $n=3$ replicates $(\mathbf{b}, \mathbf{c})$. Asterisks in $\mathbf{b}$ are compared with HeLa NC group. $* p<0.05$, $* * p<0.01, * * * p<0.001$; $\mathrm{ns}$, not significantly different (Student's $t$ test) 
Fig. 7 Schematic summary of the membrane pathway cancer mediated by TFs/miRNAs during the selection of TRCs. The membrane proteins affected by force from extracellular matrix (ECM) and lead to the change of downstream genes such as STAT3 and MYBL2.

These TFs involved in the regulation of WNT/TGF- $\beta$ / PI3K-AKT pathways and affected the expression of downstream cancer-related genes such as CCT3. CCT3, then affected the selection of TRCs

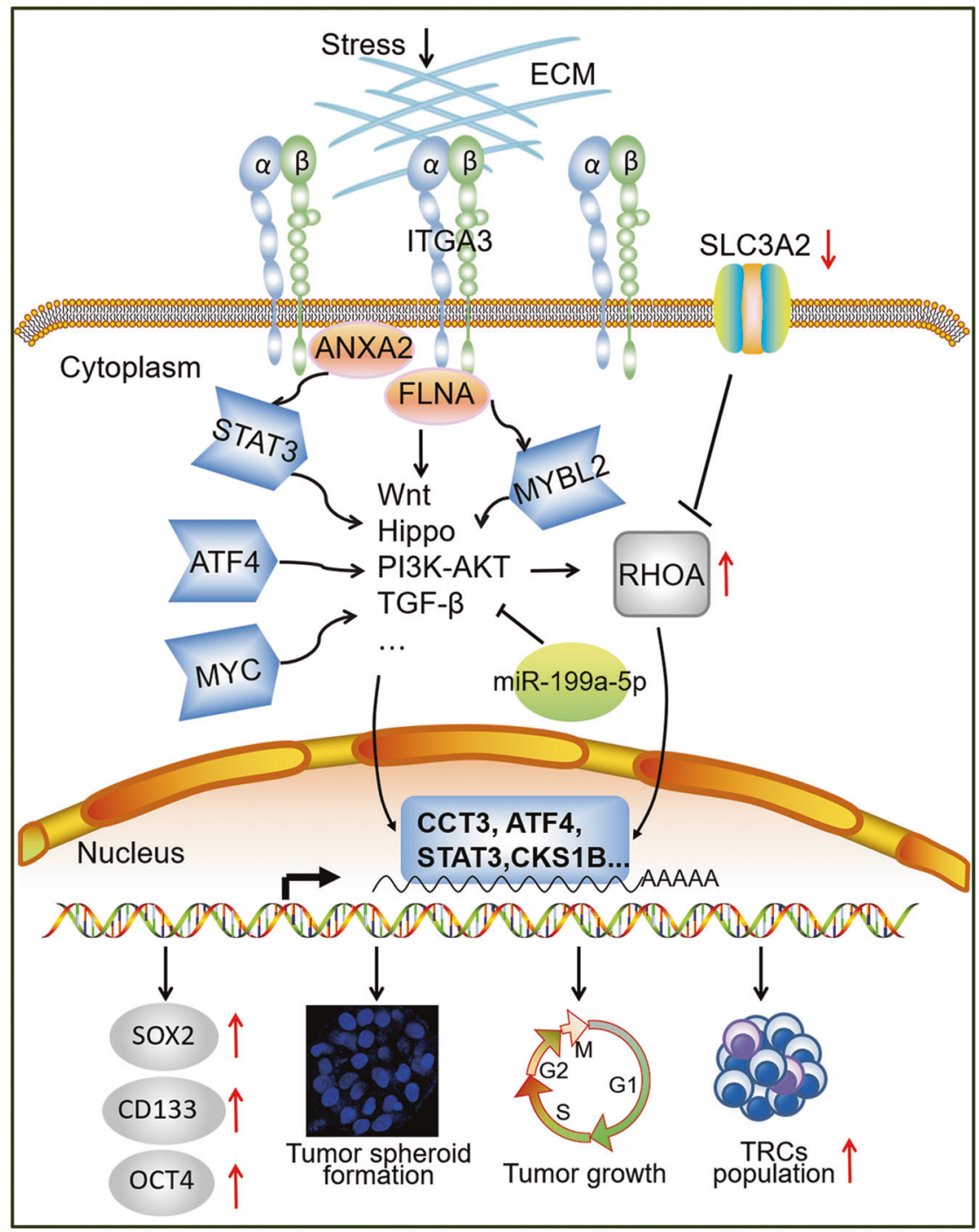

the nucleus and affect gene expression [33]. RHOA was regulated by MYC and MAZ which suggests the WNT pathway may be regulated by key TFs and participate in mechanotransduction.

We have identified seven TFs regulated the Hippo/Wnt/ TGF-beta/PI3K-AKT mechanical transduction pathways (Fig. 3b). TFs such as ATF4/MYC/STAT3 have been studied in mechanotransduction [23, 34]. MAZ can induce the differentiation of neural stem cells [35]. YBX1 can bind $N A N O G$ to affect the pluripotency of embryonic stem cells [36], and also regulates $S O X 2$, which is a self-renewal gene highly expressed in melanoma TRCs [4] and Hela TRCs, thus to maintain the stemness of breast cancer and tumorigenic properties [37]. These TFs potentially build a bridge between the mechanical transduction and cell stemness. Further multi-networks integration analyses (Fig. 4b) provided opportunities to identify key regulators/genes in the crosstalk of these functions.
The colony formation data in Fig. 4 showed that ATF4 and CCT3 promoted the TRCs selection process, while SLC3A2 and hsa-miR-199a-5p suppressed it. ATF4 as an important TF plays a pivotal role in the development of functional of mice hematopoietic stem cells [38] and relates to the drug resistance in tumor therapy [39]. ATF4 is also reported to confer a survival advantage for tumor cells under hypoxia and regulate tumor progression [40]. In accordance with the published reports, our results show that ATF4 promotes the generation and maintenance of TRCs and may be a tumor therapeutic target.

CCT3 is reported to promote cell proliferation and invasion of HCC cells [27] and to be regulated by STAT3 [41]. However, few studies about the CCT3 function on stemness were found. Our work identified that CCT3 may take part in the cell cycle pathway to promote proliferation of HeLa cells (Fig. 5b). MAD2L1 is an integral part of the mitotic spindle assembly checkpoint gene to ensure mitosis 
[42], and was significantly upregulated after increasing $C C T 3$, consistent with the report that CCT3 affects mitotic process [27]. CCT3 overexpression and silencing affect a large number of stemness genes (Fig. 6a) and common stemness marker genes, such as CD133, ETV4, E2F4, $K R T 8$, and SOX2 (Fig. 6c). It had been reported that the majority of SP cells showed CD133+ phenotype [43]. CCT3 overexpression cells perform both CD133+ and high SP fraction, while CCT3-silencing cells perform CD133 downregulation and low-SP fraction (Fig. 6b, c). The stemness genes were upregulated along with CCT3 upregulation in TRCs (Fig. 6d). These indicate that CCT3 may be a stemness-related gene. In addition, SLC $3 A 2$ has been reported to interact with integrins on cell membrane [44]. $S L C 3 A 2$ can activate the $R O C K$ activity through $R H O A$ (Fig. 7) and result in an increase in transcription mediated by YAP/TAZ, which is a nuclear relay signal for mechanotransduction [44]. In contrast, we found that SLC3A2 suppressed the formation and growth of TRCs (Fig. 4g), suggesting that $S L C 3 A 2$ may be an inhibitor of self-renewal and stemness of tumor cells.

Does the fibrin gel artificially promote TRCs? Whenever an assay or a probe is introduced into a biological system, there is always a possibility that this assay or probe might perturb the system and causes unwanted changes to the system. Therefore, we cannot rule out the possibility that the fibrin gels are artificially promoting TRCs. However, a published report reveals that in human malignant tumors fibrin is found in connective tissue stroma [45]. Furthermore, it is known that the survival and metastatic potential of circulating tumor cells are elevated by fibrin and fibrinogen [46]. In addition, lung metastasis is enhanced by fibrin and fibronectin complexes [47] and fibrinogen depletion reduces tumor formation in the mouse lung [48]. All these findings suggest that the soft fibrin environment is physiological relevant in tumor.

We performed GATK workflow [49] for SNP and indel calling on RNA-seq data of HeLa $2 \mathrm{D}$ sample ( $0 \mathrm{~h}$ sample), and four TRCs samples $(12 \mathrm{~h}, 1$ day, 3 day, and 5 day samples). When compared with HeLa 2D samples, we identified changes at 1065 mutation sites on 839 proteincoding genes in all TRCs samples. Almost all (97.2\%) of these mutation sites were lost in TRCs samples and only 29 mutation sites were added in TRCs samples. These suggest that more mutation sites disappear in the TRCs. However, at the present time, we do not know what the exact significance of these mutations is for TRCs when compared with $2 \mathrm{D}$ cells. To further investigate biological functions of genes with mutation changes from 2D to TRCs, we performed the KEGG pathway, GO, and stemness enrichment analyses for the 839 genes (Supplementary Fig. S8). The results showed that these genes were significantly enriched in differentiation, migration, proliferation, self-renewing, and stemness-related terms (Supplementary Fig. S8). Although a large number of genes were associated with cancer growth terms (Supplementary Fig. S8), only 83 mutation sites have clinical disease-related studies, and only three mutation sites have been reported to be associated with cancer by literature search. A frameshift variant (c.4666dup, dbSNP id: rs587783031) and a missense variant (c.5465T>G, dbSNP id: rs459552) of APC gene were related with hereditary cancer-predisposing syndrome [50]. A frameshift variant (c.623dupA, dbSNP id: rs587780033) of BARD1 was found in breast cancer [51]. Clearly additional studies are needed to explore the relationship between other mutation sites and tumors.

In conclusion, in this study, we performed transcriptome data analysis and validation of TRCs from the 3D culture. We systematically investigated the gene expression alteration and regulation in mechanotransduction, including membrane protein genes, downstream mechanotransduction related signaling pathways, and cancer-related genes (Fig. 7). The current study provides us a deeper understanding of how matrix softness impacts cancer cell selection and fate via mechanotransduction pathways and regulators. This provides new insights into the CSCs initiation and cancer progression.

\section{Materials and methods}

\section{Cell lines and cell culture}

Human cervical cancer cell line HeLa was kept by our laboratory and had STR tested by CCTCC (Supplementary Table S2). Human hepatocarcinoma cell line Bel7402 was purchased from China Center for Type Culture Collection (CCTCC, Wuhan, China). Cells were cultured on the plastic plate in DMEM (high glucose Dulbecco's modified Eagle's medium) with $10 \%$ fetal bovine serum (Life Technologies, Carlsbad, CA, USA), and $100 \mathrm{mg} / \mathrm{ml}$ penicillin/streptomycin at $37{ }^{\circ} \mathrm{C}$ with $5 \% \mathrm{CO}_{2}$. The cells were randomly assigned to each experimental group and the potential presence of mycoplasma was monitored via continuous microscopic imaging.

\section{HeLa 3D preparation}

HeLa cells were digested with trypsin (Life Technologies, Carlsbad, CA, USA) from the 2D plastic plate, then were divided into single-cell solution. Fibrinogen was diluted into 2, 8 , or $16 \mathrm{mg} / \mathrm{ml}$ with $\mathrm{T} 7$ buffer $(\mathrm{pH} 7.4,50 \mathrm{mM}$ Tris, and $150 \mathrm{mM} \mathrm{NaCl}$ ). Cell plus fibrinogen mixture was made by mix the same volume of single-cell solution and fibrinogen solution. Finally, the mixture's concentration [4] is 1,4 , or $8 \mathrm{mg} / \mathrm{ml}$ fibrin gels (corresponding stiffness is 90, 420, and $1050 \mathrm{~Pa}$, respectively). For colony number experiment, $50 \mu \mathrm{l}$ cell and fibrinogen mixtures were seeded into each well of 96- 
well plate and mixed well with preadded $1 \mu$ thrombin $(100 \mathrm{U} / \mathrm{ml})$. And $250 \mu \mathrm{l}$ cell plus fibrinogen mixture was seeded into each well of 24-well plate with $5 \mu$ l thrombin for total RNA extraction. The cell culture plate was then incubated in $37^{\circ} \mathrm{C}$ cell culture incubator for $25 \mathrm{~min}$. Finally, DMEM with $10 \%$ fetal bovine serum and antibiotics was added with quadruple volume of the mixture.

\section{Real-time qPCR analysis}

Cells were lysed with TRIzol ${ }^{\mathrm{TM}}$ reagent (Life Technologies, Carlsbad, CA, USA), and total RNA was isolated according to the TRIzol ${ }^{\mathrm{TM}}$ Reagent User Guide (https://www. thermofisher.com/). Reverse transcription was performed using the TransScript First-strand cDNA Synthesis Super Mix (TransGen Biotech, Beijing, China), according to the manufacturer's instructions. Real-time quantitative PCR (qPCR) was performed using GoTaq qPCR Master Mix (Promega, Madison, MI, USA). All mRNA expression was normalized to human glyceraldehyde 3-phosphate dehydrogenase (GAPDH). Sequences of all the primers for realtime qPCR are listed in Supplementary Table S3. All primers were designed by Primer Premier 6, and synthesis by TSINGKE (Beijing, China).

The expression of miRNA was detected by All-in-One ${ }^{\mathrm{TM}}$ miRNA qRT-PCR Detection kit (GeneCopoeia ${ }^{\mathrm{TM}}$, Guangzhou, China) according to the manufacturer's instructions. All miRNA expression was normalized to hsa-U6.

\section{Transfection}

Cells were transfected with small-interfering RNA (siRNA) using Lipofectamine 2000 (Life Technologies, Carlsbad, CA, USA) as manufacturer's instructions. The NC siRNA, siRNA duplexes and shRNA were synthesized from Genephama Biotech (Shanghai, China). All the siRNA sequences are listed in Supplementary Table S4, all shRNA sequences are listed in Supplementary Table S5. Silence efficiencies were shown in Supplementary Figs. S5D and 6A. CCT3 expression construct was generated by subcloning PCR-amplified full-length human CCT3 cDNA into the pcDNA3.1 plasmid.

Hsa-miR-199a-5p mimic, specific inhibitor molecules, and appropriate NC molecules were purchased from Genephama Biotech (Shanghai, China). All the mimic, inhibitor or NC molecules were transfected using X-treme GENE siRNA Transfection Reagent (Roche, Germany), based on the manufacturer's protocol.

\section{Colony number assay and colony size calculate}

By changing the focal planes according to the direction of gel depth, the colony number was counted view by view using optical microscope (MDIL-LED, Leica, Germany). At least three wells of colonies were counted per condition. The colony photograph was taken by using an inverted fluorescence microscope (DMI6000B, Leica, German). The area $(S)$ of each colony was measured by using ImageJ, and then the size $(V)$ was calculated by using the formula: $V=\frac{4}{3} \times \sqrt{\left(s^{3} / \pi\right)}$.

\section{Migration and invasion assay}

HeLa 3D was digested by collagenase and dispase II mixture in $37^{\circ} \mathrm{C}$ to dissolve the fibrin gels and divided the colonies into the single-cell suspension. For the migration assay, $4 \times 10^{4}$ cells in serum-free medium were seed in the upper compartment of an $8 \mu \mathrm{m}$ transwell chamber (Corning, NY, USA). For the invasion assay, the upper chambers were previously coated with ECM gel (Corning, NY, USA). After incubation for $24-48 \mathrm{~h}$, the migrated or invaded cells on the lower membrane were stained with $4 \%$ paraformaldehyde, then stained with $0.1 \%$ crystal violet. The upper cells were moved gently by the soft medical cotton ball. Each group random took ten pictures under a microscope with $\times 400$ magnification (Olympus, Japan). Cell numbers per field were calculated by ImageJ.

\section{Data source}

HeLa cells cultured in the rigid plastic plate were marked as HeLa 2D, and in 90 Pa fibrin gels were marked as HeLa 3D. The cells before cultured into fibrin gels were collected with TRIzol $^{\mathrm{TM}}$ Regent as $0 \mathrm{~h}$. HeLa 3D cells were collected with TRIzol Regent in a row at four time points: $12 \mathrm{~h}, 1$ day (24 h), 3 day (72 h), and 5 day (120 h). All samples have carried out high-throughput sequencing: RNA-Seq (PE150) and miRNA-Seq (SE50) via Illumina HiSeqTM4000 platform by BGI-shenzhen (Wuhan, China). We obtained 12.5 million clean reads for miRNA and 80 million clean reads for RNA of each sample. RNA-Seq reads were mapped to Ensembl v81 (GRCH38) and processed by the HISAT2StringTie-ballgown pipeline [52]. miRNA-Seq reads were to map the miRNA precursor sequence to get known miRNA expression based on our previous pipeline [53]. We used FPKM (Fragments Per Kilobase per Million mapped reads) and RPM (Reads Per Million) to calculate gene and miRNA expression levels, separately.

\section{Differentially expressed genes}

We used bioconductor-package NOISeq [54] to screen DEGs or DEMs. Differentiation analysis was performed on genes or miRNAs that expressed more than 20 (FPKM/ RPM) in any sample. Genes or miRNAs with prob $>0.99$ and IFold Changel $\geq 2$ were considered as significant DEGs/ 
DEMs. Differentiation analysis of CCT3 overexpression or CCT3 RNAi compare with control were also performed by NOISeq with cutoff prob $>0.7$ and IFold Changel $\geq 1.5$. All of the heatmaps were visualized by software MeV (http:// en.bio-soft.net/chip/MeV.html). All genes were normalized by row and hierarchical clustering was performed by calculating Euclidean distance.

\section{Quantification of continuously altered genes}

Because of the drastic changes in gene expression between HeLa 2D and HeLa 3D, we just considered the genes whose expression levels continuously increased or decreased from $12 \mathrm{~h}$ to 5 day and the fold change of 5 day versus $12 \mathrm{~h}$ was larger than 1.5 times as continuously increased/decreased gene. miRNAs whose expression continuously increased/ decreased from $0 \mathrm{~h}$ to 3 day and the fold change of 3 day versus $0 \mathrm{~h}$ was higher than 1.5 times were considered as continuously increased or decreased miRNAs. We collected the stemness genes/miRNAs from pieces of literature and intersected them with continuously altered genes or miRNAs for further analysis.

\section{Enrichment and stemness significance analysis}

GO and KEGG enrichment analyses of all DEGs were achieved by online tool DAVID [55] (https://david.ncifcrf. gov/) (FDR $\leq 0.01)$. KEGG enrichment analysis for genes in the integrated network in Fig. 6c was based on the Cytoscape plug-in ClueGO (http://apps.cytoscape.org/apps/ cluego) [56] $(P \leq 0.01)$. The stemness enrichment analyses were carried out by StemChecker database (http:// stemchecker.sysbiolab.eu/, $P \leq 0.01)$.

\section{Regulatory networks}

Construction of regulatory networks utilizes interaction data collected in the previous work [13], including experimental and predicted TF-target/miRNA and miRNA-target/TF. We constructed the membrane protein network using differentially expressed membrane genes, differentially expressed TFs and 29 key DEMs, which are at the intersection of 3D vs $2 \mathrm{D}$ different miRNAs and continuously altered miRNAs. Combined the differentially expressed TFs (Fig. 1g), DEGs (differed between $0 \mathrm{~h}$ (HeLa 2D) and $12 \mathrm{~h}$ (HeLa 3D)), and key DEMs, we constructed the four classical pathway networks in the KEGG pathway (http://www.kegg.jp/kegg/).

\section{Variants identification}

In this study, we used the best practices workflow by GATK [49] to detect SNP and indel on RNA-Seq data. First, we used STAR 2-pass alignment with the default parameters mapping to Ensembl v81 (GRCH38) and used Picard to add group information to the mapping result SAM files. Then, we used GATK tool (version 3.7) [49] to split "N" Trim and reassign-mapping qualities. Finally, we used the recommended HaplotypeCaller in GATK to perform variant calling and used ANNOVAR [57] to annotate mutations.

\section{Data and software availability}

Sequencing data in this study were deposited at the BIG Data Center with the accession code (CRA000751), which can be accessed publicly via http://bigd.big.ac.cn/gsa.

\section{Cell proliferation assay}

Cell proliferation was measured with the Cell-Counting Kit8 (CCK-8) assay kit (Dojindo Corp., Japan). HeLa 2D cells or Bel7402 2D cells were seeded in 96-well plates $\left(8 \times 10^{3}\right.$ cells/well). Empty vectors, pcDNA 3.1-CCT3 or RNAi were transfected for $24 \mathrm{~h}$ (HeLa) and $48 \mathrm{~h}$ (Bel7402). Ten microliters CCK-8 reagent was added to each well. After incubating for $4 \mathrm{~h}$, the cell density was measured indirectly through quantification of the solubilized formazan product at $450 \mathrm{~nm}$ with Flexstation $3^{\circledR}$ microplate reader (Molecular Device, CA, US). Three independent experiments were performed.

\section{Side population analyses}

Cells were harvested after transfected with vectors for $24 \mathrm{~h}$, washed with PBS. Then cells were stained with $5 \mu \mathrm{g} / \mathrm{ml}$ Hoechst $33342 \pm 50 \mu \mathrm{M}$ verapamil for $90 \mathrm{~min}$ at $37^{\circ} \mathrm{C}$. At the end of incubation, cells were centrifuged at $4{ }^{\circ} \mathrm{C}$ and washed by cold PBS once. SP cells and non-SP cells were differentiated by a flow cytometry-assisted cell sorter (Cytoflex, Beckman, USA). At least 50,000 events were examined [58].

\section{Western blotting assay}

Cells and tumor tissues were lysed with RIPA Lysis buffer (Beyotime, China), protein concentrations were determined by the BCA kit (Beyotime, China). Each sample was separated by $8-12 \%$ SDS-PAGE, blocked with $5 \%$ BSA for $2 \mathrm{~h}$ at room temperature and incubated overnight at $4{ }^{\circ} \mathrm{C}$ with primary antibodies: CCT3 Polyclonal Antibody (A6547, ABclonal, China), SOX2 Polyclonal Antibody (A0561, ABclonal, China), KRT8 Polyclonal Antibody (A1024, ABclonal, China), CD133 Polyclonal Antibody (A12711, ABclonal, China), GAPDH Polyclonal Antibody (AC001, ABclonal, China), ETV4 Polyclonal Antibody (A5797, ABclonal, China), and E2F4 Antibody (10923-1AP, Proteintech, China). Primary antibodies were detected 
with goat anti-Rabbit IgG-HRP (sc-2004, Santa Cruz, TX, USA) or anti-Mouse IgG-HRP (sc-2005, Santa Cruz, TX, USA). The blots were developed using Super Signal West Pico chemiluminescent substrate (Millipore, MA, USA).

\section{Animals}

Four-week-old BALB/c nude female mice and male mice were obtained from (Beijing HFK, China). The mice were randomly assigned to be in the control group or the treated group, each group had eight mice. The experimentalists were blinded from the expected outcome of the treatment. All animals received humane care in compliance with the Principles of Laboratory Animal Care Formulated by the National Society of Medical Research and the guide for the US National Institutes of Health. The protocol was approved by the Animal Care and Use Committee of Huazhong University of Science and Technology.

\section{Immunofluorescence}

Cells were fixed with $4 \%$ formaldehyde (BioLegend, China) for $20 \mathrm{~min}$ at room temperature, washed with PBS thrice. Then permeabilized with $0.2 \%$ Triton X-100 (BioLegend, China) for $20 \mathrm{~min}$ and blocked with $10 \%$ donkey serum (Solarbio, China) $4 \mathrm{~h}$. Incubated primary antibody, antiCCT3 (A6547, ABclonal, China) overnight at $4{ }^{\circ} \mathrm{C}$. After washed with PBS thrice, the secondary antibody Alexa Fluor $R 488$ (A11034, Invitrogen, USA) and DAPI (Biosharp, China) were incubated for $2 \mathrm{~h}$ avoid light. Images were acquired with inverted fluorescence microscope (DMI6000B, Leica, German). Relative intensity was calculated by ImageJ.

\section{Statistical analysis}

Statistical analyses were performed using GraphPad Prism 7.0 and R package. Data are represented as the mean \pm s.e. $\mathrm{m}$. from at least three independent experiments. Comparisons between two groups were performed using Student's $t$ test, and single, double and triple asterisks indicating statistical significance: $* p<0.05, * * p<0.01, * * * p<0.001$, while ns indicates not significantly different.

Acknowledgements We acknowledge the research funding from National Basic Research Program of China (2015CB931802), National Natural Science Foundation of China (NSFC) [81627901, 81773653, 31822030, and 31771458], and Program for Changjiang Scholars and Innovative Research Team in University (PCSIRT) [No. IRT 13016].

\section{Compliance with ethical standards}

Conflict of interest The authors declare that they have no conflict of interest.
Publisher's note: Springer Nature remains neutral with regard to jurisdictional claims in published maps and institutional affiliations.

Open Access This article is licensed under a Creative Commons Attribution 4.0 International License, which permits use, sharing, adaptation, distribution and reproduction in any medium or format, as long as you give appropriate credit to the original author(s) and the source, provide a link to the Creative Commons license, and indicate if changes were made. The images or other third party material in this article are included in the article's Creative Commons license, unless indicated otherwise in a credit line to the material. If material is not included in the article's Creative Commons license and your intended use is not permitted by statutory regulation or exceeds the permitted use, you will need to obtain permission directly from the copyright holder. To view a copy of this license, visit http://creativecommons. org/licenses/by/4.0/.

\section{References}

1. Hanahan D, Weinberg RA. Hallmarks of cancer: the next generation. Cell. 2011;144:646-74.

2. Najafi M, Farhood B, Mortezaee K. Cancer stem cells (CSCs) in cancer progression and therapy. J Cell Physiol. 2019;234:8381-95.

3. Nimmakayala RK, Batra SK, Ponnusamy MP. Unraveling the journey of cancer stem cells from origin to metastasis. Biochim Biophys Acta Rev Cancer. 2018;1871:50-63.

4. Liu J, Tan Y, Zhang H, Zhang Y, Xu P, Chen J, et al. Soft fibrin gels promote selection and growth of tumorigenic cells. Nat Mater. 2012;11:734-41.

5. Tan Y, Tajik A, Chen J, Jia Q, Chowdhury F, Wang L, et al. Matrix softness regulates plasticity of tumour-repopulating cells via H3K9 demethylation and Sox2 expression. Nat Commun. 2014;5:4619.

6. Li Y, Luo S, Ma R, Liu J, Xu P, Zhang H, et al. Upregulation of cytosolic phosphoenolpyruvate carboxykinase is a critical metabolic event in melanoma cells that repopulate tumors. Cancer Res. 2015;75:1191-6.

7. Chen J, Cao X, An Q, Zhang Y, Li K, Yao W, et al. Inhibition of cancer stem cell like cells by a synthetic retinoid. Nat Commun. 2018;9:1406.

8. Ning W. Review of cellular mechanotransduction. J Phys D Appl Phys. 2017;50:233002.

9. Vining $\mathrm{KH}$, Mooney DJ. Mechanical forces direct stem cell behaviour in development and regeneration. Nat Rev Mol Cell Biol. 2017;18:728.

10. Dupont S, Morsut L, Aragona M, Enzo E, Giulitti S, Cordenonsi $\mathrm{M}$, et al. Role of YAP/TAZ in mechanotransduction. Nature. 2011;474:179.

11. Morgan JT, Murphy CJ, Russell P. What do mechanotransduction, Hippo, Wnt, and TGF $\beta$ have in common? YAP and TAZ as key orchestrating molecules in ocular health and disease. Exp Eye Res. 2013;115:1-12.

12. Takebe N, Harris PJ, Warren RQ, Ivy SP. Targeting cancer stem cells by inhibiting Wnt, Notch, and Hedgehog pathways. Nat Rev Clin Oncol. 2011;8:97-106.

13. Zhang H-M, Kuang S, Xiong X, Gao T, Liu C, Guo A-Y. Transcription factor and microRNA co-regulatory loops: important regulatory motifs in biological processes and diseases. Brief Bioinform. 2015;16:45-58.

14. Lin Y, Sibanda VL, Zhang HM, Hu H, Liu H, Guo AY. MiRNA and TF co-regulatory network analysis for the pathology and recurrence of myocardial infarction. Sci Rep. 2015;5:9653.

15. Bu P, Wang L, Chen KY, Srinivasan T, Murthy PK, Tung KL, et al. A miR-34a-Numb feedforward loop triggered by 
inflammation regulates asymmetric stem cell division in intestine and colon cancer. Cell Stem Cell. 2016;18:189-202.

16. Rysä J, Tokola H, Ruskoaho H. Mechanical stretch induced transcriptomic profiles in cardiac myocytes. Sci Rep. 2018;8 (1):4733.

17. Scherer WF, Syverton JT, Gey GO. Studies on the propagation in vitro of poliomyelitis viruses. IV. Viral multiplication in a stable strain of human malignant epithelial cells (strain HeLa) derived from an epidermoid carcinoma of the cervix. J Exp Med. 1953;97:695-710.

18. Amini S, Fathi F, Mobalegi J, Sofimajidpour H, Ghadimi T. The expressions of stem cell markers: Oct4, Nanog, Sox2, nucleostemin, Bmi, Zfx, Tcl1, Tbx3, Dppa4, and Esrrb in bladder, colon, and prostate cancer, and certain cancer cell lines. Anat Cell Biol. 2014;47:1-11.

19. Chen KD, Li YS, Kim M, Li S, Yuan S, Chien S, et al. Mechanotransduction in response to shear stress. Roles of receptor tyrosine kinases, integrins, and Shc. J Biol Chem. 1999;274:18393-400.

20. Viladiu P. The in vivo uptake of tritiated estradiol in carcinoma of the breast. Surg Gynecol Obstet. 1975;140:544-6.

21. Zhang T, Ma J, Cao X. Grb2 regulates Stat 3 activation negatively in epidermal growth factor signalling. Biochem J. 2003;376(Pt 2):457-64.

22. Wang T, Yuan J, Zhang J, Tian R, Ji W, Zhou Y, et al. Anxa2 binds to STAT3 and promotes epithelial to mesenchymal transition in breast cancer cells. Oncotarget. 2015;6:30975-92.

23. Hamzeh MT, Sridhara R, Alexander LD. Cyclic stretch-induced TGF- $\beta 1$ and fibronectin expression is mediated by $\beta 1$-integrin through c-Src- and STAT3-dependent pathways in renal epithelial cells. Am J Physiol Renal Physiol. 2015;308:F425-36.

24. Lam WA, Cao L, Umesh V, Keung AJ, Sen S, Kumar S. Extracellular matrix rigidity modulates neuroblastoma cell differentiation and $\mathrm{N}$-myc expression. Mol Cancer. 2010;9:35.

25. Luo Y, Xu X, An X, Sun X, Wang S, Zhu D. Targeted inhibition of the miR-199a/214 cluster by CRISPR interference augments the tumor tropism of human induced pluripotent stem cell-derived neural stem cells under hypoxic condition. Stem Cells Int. 2016;2016:3598542.

26. Zheng J, Li X-d, Wang P, Liu X-b, Xue Y-x, Hu Y, et al. CRNDE affects the malignant biological characteristics of human glioma stem cells by negatively regulating miR-186. Oncotarget. 2015;6:25339-55.

27. Zhang Y, Wang Y, Wei Y, Wu J, Zhang $\mathrm{P}$, Shen $\mathrm{S}$, et al. Molecular chaperone CCT3 supports proper mitotic progression and cell proliferation in hepatocellular carcinoma cells. Cancer Lett. 2016;372:101-9.

28. Mobus S, Yang D, Yuan Q, Ludtke TH, Balakrishnan A, Sgodda M, et al. MicroRNA-199a-5p inhibition enhances the liver repopulation ability of human embryonic stem cell-derived hepatic cells. J Hepatol. 2015;62:101-10.

29. Koshizuka K, Hanazawa T, Kikkawa N, Arai T, Okato A, Kurozumi A, et al. Regulation of ITGA3 by the anti-tumor miR-199 family inhibits cancer cell migration and invasion in head and neck cancer. Cancer Sci. 2017;108:1681-92.

30. Cui X, Hu Z-P, Li Z, Gao P-J, Zhu J-Y. Overexpression of chaperonin containing TCP1, subunit 3 predicts poor prognosis in hepatocellular carcinoma. World J Gastroenterol. 2015;21:8588-604.

31. Shafer B, Onishi K, Lo C, Colakoglu G, Zou Y. Vang12 promotes Wnt/planar cell polarity-like signaling by antagonizing Dvl1mediated feedback inhibition in growth cone guidance. Dev Cell. 2011;20:177-91.

32. Habas R, Kato Y, He X. Wnt/Frizzled activation of Rho regulates vertebrate gastrulation and requires a novel formin homology protein Daam1. Cell. 2001;107:843-54.
33. Wozniak MA, Chen CS. Mechanotransduction in development: a growing role for contractility. Nat Rev Mol Cell Biol. 2009;10:34-43.

34. Sironen RK, Karjalainen HM, Törrönen K, Elo MA, Kaarniranta $\mathrm{K}$, Takigawa M, et al. High pressure effects on cellular expression profile and mRNA stability. A cDNA array analysis. Biorheology. 2002;39:111-7.

35. Wang J, Cheng H, Li X, Lu W, Wang K, Wen T. Regulation of neural stem cell differentiation by transcription factors HNF4-1 and MAZ-1. Mol Neurobiol. 2013;47:228-40.

36. Guo C, Xue Y, Yang G, Yin S, Shi W, Cheng Y, et al. Nanog RNA-binding proteins YBX1 and ILF3 affect pluripotency of embryonic stem cells. Cell Biol Int. 2016;40:847-60.

37. Jung K, Wu F, Wang P, Ye X, Abdulkarim BS, Lai R. YB-1 regulates Sox 2 to coordinately sustain stemness and tumorigenic properties in a phenotypically distinct subset of breast cancer cells. BMC Cancer. 2014;14:328.

38. Zhao Y, Zhou J, Liu D, Dong F, Cheng H, Wang W, et al. ATF4 plays a pivotal role in the development of functional hematopoietic stem cells in mouse fetal liver. Blood. 2015;126:2383-91.

39. Matsumoto T, Uchiumi T, Monji K, Yagi M, Setoyama D, Amamoto R, et al. Doxycycline induces apoptosis via ER stress selectively to cells with a cancer stem cell-like properties: importance of stem cell plasticity. Oncogenesis. 2017;6:397.

40. Xia HF, Zhu JY, Wang JN, Ren JG, Cai Y, Wang FQ, et al. Association of ATF4 expression with tissue hypoxia and M2 macrophage infiltration in infantile hemangioma. J Histochem Cytochem. 2017;65:285-94.

41. Shi L, Wang SQ, Zangari M, Xu HW, Cao TM, Xu CJ, et al. Over-expression of CKS1B activates both MEK/ERK and JAK/ STAT3 signaling pathways and promotes myeloma cell drugresistance. Oncotarget. 2010;1:22-33.

42. López-Saavedra A, Ramírez-Otero M, Díaz-Chávez J, CáceresGutiérrez R, Justo-Garrido M, Andonegui MA, et al. MAD2 $\gamma$, a novel MAD2 isoform, reduces mitotic arrest and is associated with resistance in testicular germ cell tumors. Cell Cycle. 2016;15:2066-76.

43. Sun H, Hou H, Lu P, Zhang L, Zhao F, Ge C, et al. Isocorydine inhibits cell proliferation in hepatocellular carcinoma cell lines by inducing G2/M cell cycle arrest and apoptosis. PLoS ONE. 2012;7:e36808.

44. Estrach S, Lee S-A, Boulter E, Pisano S, Errante A, Tissot FS, et al. CD98hc (SLC3A2) loss protects against ras-driven tumorigenesis by modulating integrin-mediated mechanotransduction. Cancer Res. 2014;74:6878-89.

45. Costantini V, Zacharski LR. The role of fibrin in tumor metastasis. Cancer Metastasis Rev. 1992;11:283-90.

46. Palumbo JS, Degen JL. Fibrinogen and tumor cell metastasis. Haemostasis. 2001;31(Suppl 1):11-5.

47. Malik G, Knowles LM, Dhir R, Xu S, Yang S, Ruoslahti E, et al. Plasma fibronectin promotes lung metastasis by contributions to fibrin clots and tumor cell invasion. Cancer Res. 2010;70:4327-34.

48. van Sluis GL, Bruggemann LW, Esmon CT, Kamphuisen PW, Richel DJ, Buller HR, et al. Endogenous activated protein $\mathrm{C}$ is essential for immune-mediated cancer cell elimination from the circulation. Cancer Lett. 2011;306:106-10.

49. McKenna A, Hanna M, Banks E, Sivachenko A, Cibulskis K, Kernytsky A, et al. The Genome Analysis Toolkit: A MapReduce framework for analyzing next-generation DNA sequencing data. Genome Res. 2010;20:1297-303.

50. Rivera B, González S, Sánchez-Tomé E, Blanco I, Mercadillo F, Letón R, et al. Clinical and genetic characterization of classical forms of familial adenomatous polyposis: a Spanish population study. Ann Oncol. 2011;22:903-9.

51. Susswein LR, Marshall ML, Nusbaum R, Vogel Postula KJ, Weissman SM, Yackowski L, et al. Pathogenic and likely 
pathogenic variant prevalence among the first 10,000 patients referred for next-generation cancer panel testing. Genet Med. 2015;18:823-32.

52. Pertea M, Kim D, Pertea GM, Leek JT, Salzberg SL. Transcriptlevel expression analysis of RNA-seq experiments with HISAT, StringTie and Ballgown. Nat Protoc. 2016;11:1650-67.

53. Gong J, Wu Y, Zhang X, Liao Y, Sibanda VL, Liu W, et al. Comprehensive analysis of human small RNA sequencing data provides insights into expression profiles and miRNA editing. RNA Biol. 2014;11:1375-85.

54. Tarazona S, Furió-Tarí $P$, Turrà D, Pietro AD, Nueda MJ, Ferrer A, et al. Data quality aware analysis of differential expression in RNA-seq with NOISeq R/Bioc package. Nucleic Acids Res. 2015;43:e140.
55. Huang DW, Sherman BT, Lempicki RA. Systematic and integrative analysis of large gene lists using DAVID bioinformatics resources. Nat Protoc. 2009;4:44-57.

56. Bindea G, Mlecnik B, Hackl H, Charoentong P, Tosolini M, Kirilovsky A, et al. ClueGO: a Cytoscape plug-in to decipher functionally grouped gene ontology and pathway annotation networks. Bioinformatics. 2009;25:1091-3.

57. Wang K, Li M, Hakonarson H. ANNOVAR: functional annotation of genetic variants from high-throughput sequencing data. Nucleic Acids Res. 2010;38:e164.

58. Addla SK, Brown MD, Hart CA, Ramani VAC, Clarke NW. Characterization of the Hoechst 33342 side population from normal and malignant human renal epithelial cells. Am J Physiol Ren Physiol. 2008;295:F680-7. 